Supporting Information

\title{
Zr-Based Metal-Organic Framework/Reduced Graphene Oxide Composites for Catalytic Synthesis of 2,3-Dihydroquinazolin-4(1H)-One Derivatives
}

\author{
Gyanendra Kumar ${ }^{1}$, Navin Kumar Mogha ${ }^{1,2}$ and Dhanraj T. Masram ${ }^{1 *}$ \\ ${ }^{1}$ Department of Chemistry, University of Delhi, Delhi-110007, India \\ ${ }^{2}$ Shriram Institute for Industrial Research, Delhi-110007, India
}

\section{TABLE OF CONTENTS}

1. Molecular docking study for all synthesized compounds

2. Materials and Method

3. Table of docking score (G12, G13, G14 and EB)

4. Gas adsorption performance

5. Energy Dispersive X-ray Spectroscopy (EDS)

S-6

6. Recyclability

S-6

7. Characterization of the corresponding products $\left({ }^{1} \mathrm{H},{ }^{13} \mathrm{C}\right)$ 


\section{Molecular docking}

All fourteen compounds were drawn with CS ChemDraw and saved in an appropriate format. Geometry optimization was performed with the help of Chem 3D using molecular mechanics (MM2) as a force field. Energy minimization was performed, in which the Root mean square deviation (RMS) Gradient is set to 0.010 . These optimized compounds were used in docking. The preparation of calf-thymus DNA (CT-DNA) was performed using Molegro Molecular Viewer (MMV 2.5). Where assigning of bonds, missing bond order, and hybridization, missing explicit hydrogen, missing charges, flexible torsion in compounds, tripos type atoms were checked in it. Finally, the prepared CT-DNA was used for molecular docking. Molecular docking uses the atomic label interaction to define the interaction between the small drug molecule and macromolecules like protein and nucleic acids.

Table S1. Showing molecular docking study of all compounds.

\begin{tabular}{|c|c|c|c|c|}
\hline Entry & Compound & $\begin{array}{l}\text { Binding } \\
\text { Affinity }\end{array}$ & RMSD/ub & RMSD/lb \\
\hline G1 & & -7.2 & 0 & 0 \\
\hline G2 & & & & \\
\hline G3 & & -7.6 & 0 & 0 \\
\hline G4 & & -7.4 & 0 & 0 \\
\hline G5 & & -7.5 & 0 & 0 \\
\hline
\end{tabular}




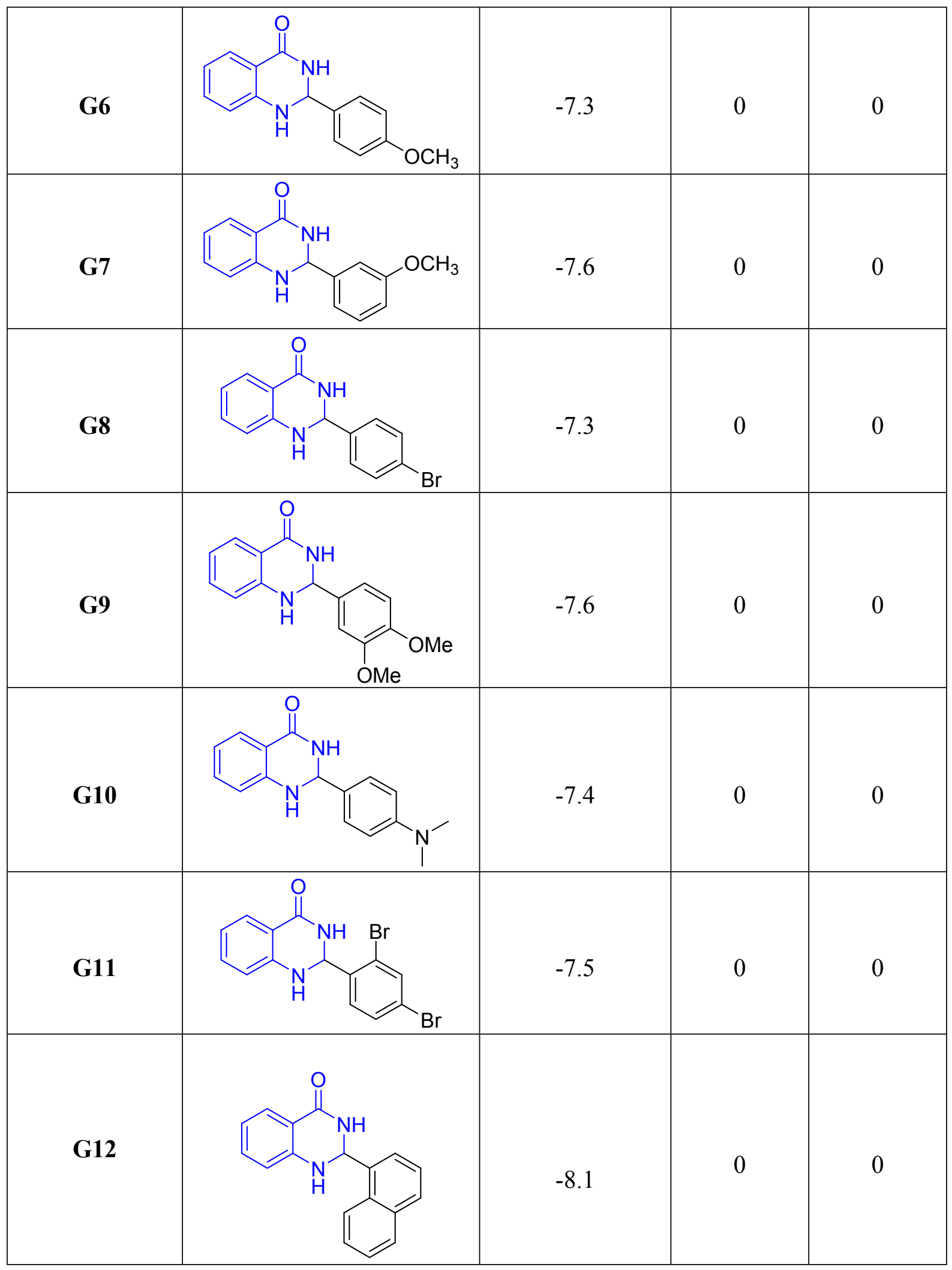

S-3 


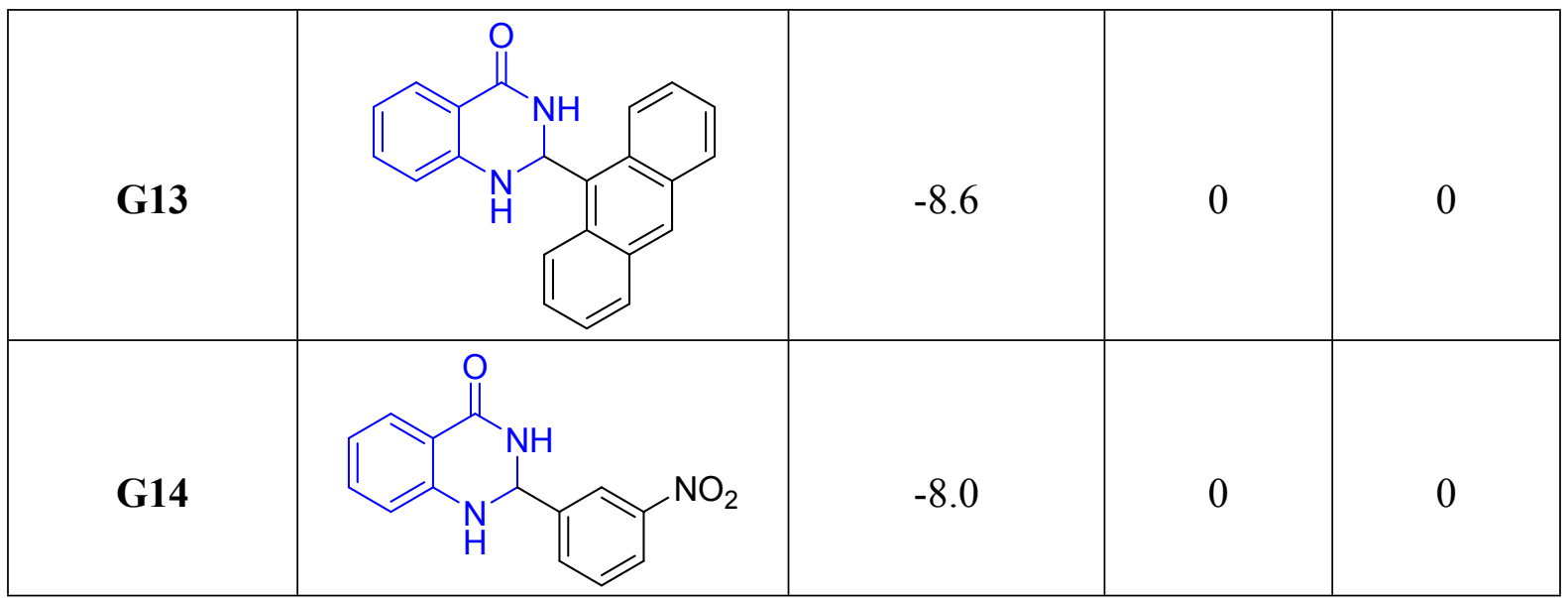

$\mathbf{R M S D} / \mathbf{u b}=$ Root mean square deviation/upper bound, RMSD/lb = Root mean square deviation/upper bound

\section{Materials and Method}

The synthesized zirconium-based metal-organic framework/reduced graphene oxide (Zr-MOF/rGO) nanocatalyst was characterized by a series of analytical techniques such as Powder X-ray diffraction results were obtained using a Shimadzu D8 DISCOVER X-ray diffractometer was used.

PHI 5000 Versa Prob II, FEI Inc. with Auger electron spectroscopy module was used for obtaining the X-ray photoelectron spectroscopy (XPS) spectra. A Morphological characterization experiment was carried out using TECNAI $200 \mathrm{kV}$ Transmission Electron Microscopy (TEM) with UHV FEI, Germany (Electron Optics) equipped through digital imaging and a $35 \mathrm{~mm}$ photography system, and Field Emission Scanning Electron Microscopy (FESEM) instruments with digital imaging and ZEISS Gemini SEM-500. Perkin Elmer TG/DTA instrument was used to perform (HiRes1000 RT to 1100C) the TGA experiment under $\mathrm{N}_{2}$ atmosphere. Raman spectra were obtained from Renishaw in Via ${ }^{\mathrm{TM}}$ Reflex MicroRaman spectrometer using $\mathrm{Ar}^{+}$laser with $514 \mathrm{~nm}$ wavelength excitation. ASI-CT-11 Quanta chrome Instrument was used for $\mathrm{N}_{2}$ Adsorption-desorption analysis was performed with Pore size distribution was determined using NLDFT. ${ }^{1} \mathrm{H}$ and ${ }^{13} \mathrm{C}$-NMR were performed on Jeol ECX $400 \mathrm{MHz}$ spectrophotometer. Fourier-transform infrared (FT-IR) spectroscopy was carried out by Thermo Scientific INCOLET iS50 spectrometer. Loading of zirconium (Zr) content was also confirmed from Inductively Coupled Plasma Optical Emission Spectroscopy (ICP-OES) performed on Thermo Scientific, iCAP7400 DUMFC.

\section{Table of docking score (G12, G13, G14 \& EB)}


Table: S2. Docking score of G12, G13, G14 \& EB.

Compounds Binding Score Root mean square deviation (RMSD) of docking

\begin{tabular}{l|lll}
\hline & & RMSD (l.b.) & RMSD (u.b.) \\
\hline $\boldsymbol{G 1 2}$ & -8.1 & 0 & 0 \\
$\boldsymbol{G 1 3}$ & -8.6 & 0 & 0 \\
$\boldsymbol{G 1 4}$ & -8.0 & 0 & 0 \\
$\boldsymbol{E B}$ & -7.4 & 0 & 0 \\
\hline
\end{tabular}

(l.b. = lower bound u.b. = upper bound)

4. Gas adsorption performance
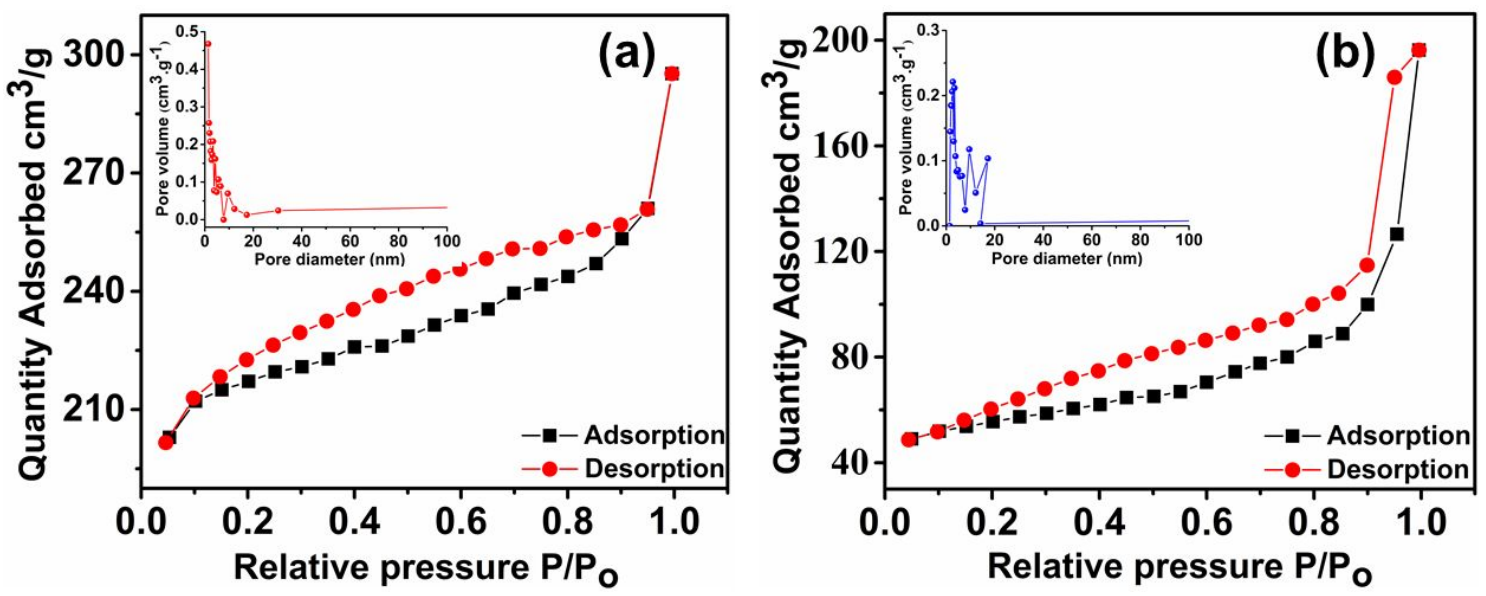

Figure: S1. BET adsorption/desorption of (a) Zr-MOF, (b) Zr-MOF/rGO-nanocatalyst, respectively.

Table: S3. The Surface area and pore size of Zr-MOF and Zr-MOF/rGO-nanocatalyst.

\begin{tabular}{|c|c|c|c|}
\hline Sample & Surface Area $\left(\mathrm{m}^{2} / \mathrm{g}\right)$ & Pore size $(\mathrm{nm})$ & Pore volume $\left(\mathrm{cm}^{3} / \mathrm{g}\right)$ \\
\hline $\mathrm{Zr}-\mathrm{MOF}$ & 634 & 2.888 & 0.3043 \\
\hline $\begin{array}{c}\mathrm{Zr}-\mathrm{MOF} / \mathrm{rGO}- \\
\text { nanocatalyst }\end{array}$ & 172.4 & 7.06 & 0.4578 \\
\hline
\end{tabular}




\section{Energy Dispersive X-ray Spectroscopy (EDS)}

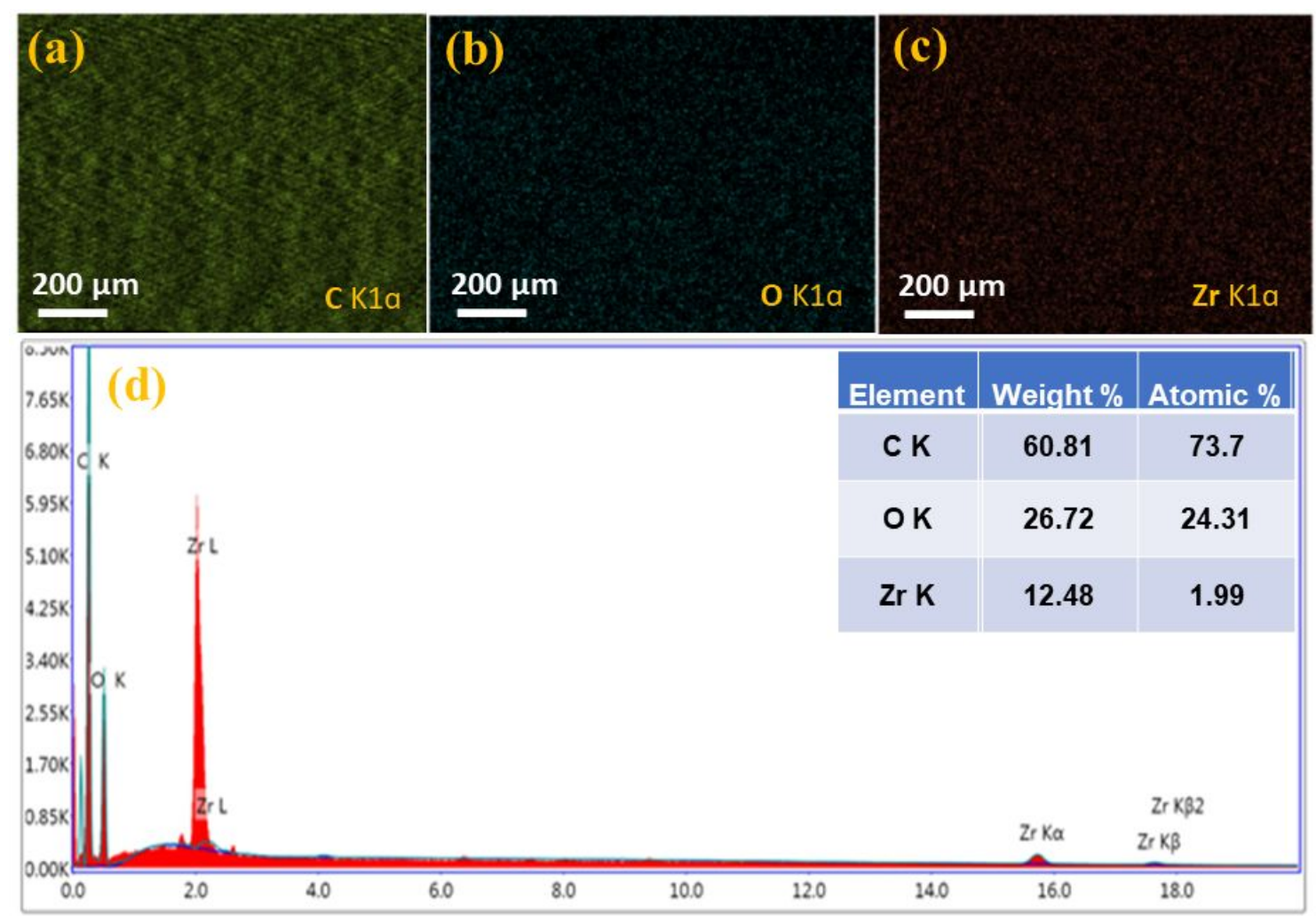

Lsec: 200.00 Cnts 0.000 keV Det: Octane Plus Det

Figure: S2. Energy Dispersive X-ray Spectroscopy images of (a) carbon, (b) oxygen, (c) zirconium (d) EDS spectra of Zr-MOF/rGO-nanocatalyst.

\section{Recyclability}

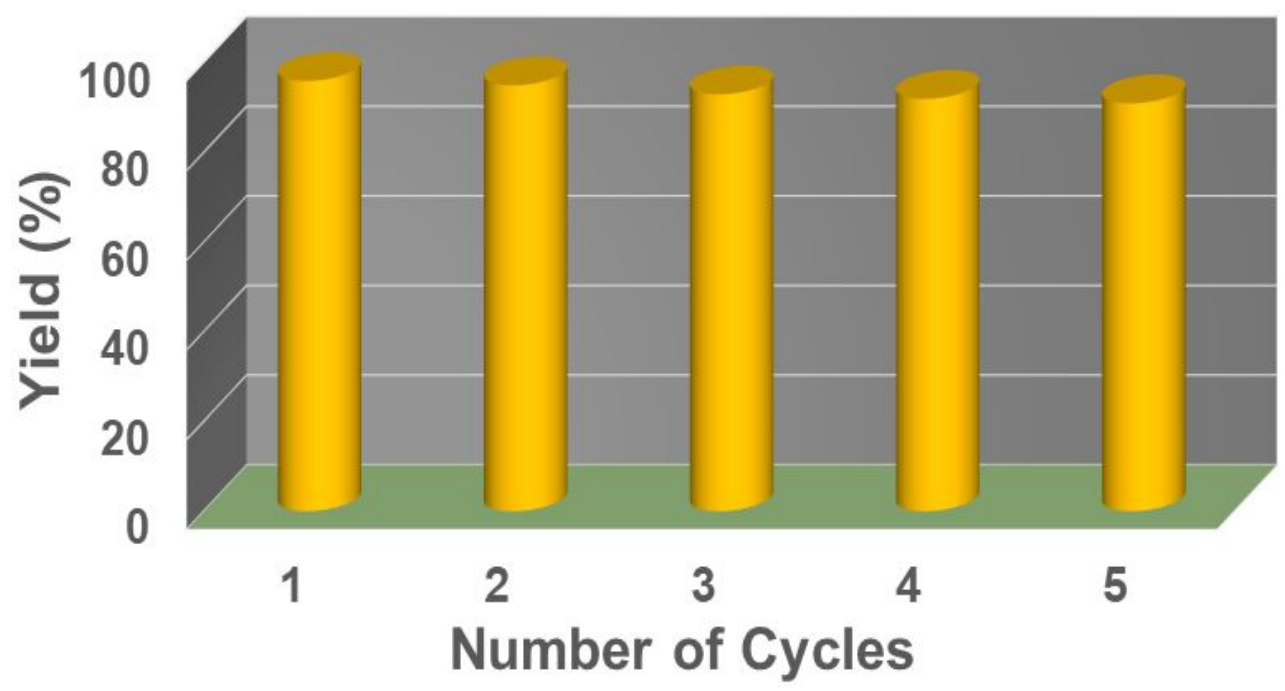

Reaction conditions: 2-aminobenzamide ( $1 \mathrm{mmol})$, Benzaldehyde $(1 \mathrm{mmol})$, nanocatalyst $(20 \mathrm{mg}), \mathrm{EtOH}$ (10 $\mathrm{mL})$, Isolated Yields.

Figure S3. Recyclability of the Zr-MOF/rGO-nanocatalyst. 


\section{Data of ${ }^{1} \mathrm{H}$ NMR, ${ }^{13} \mathrm{C}$ NMR}

\section{Compound G1.}

${ }^{1} \mathrm{H}$ NMR (400 MHz, DMSO-d 6 , ppm) $\delta: 8.30(\mathrm{~s}, J H H=8 \mathrm{~Hz}, 1 \mathrm{H}), 7.50-7.48(\mathrm{~m}$, $2 \mathrm{H}), 7.41-7.34(\mathrm{~m}, 3 \mathrm{H}), 7.22(\mathrm{t}, \mathrm{JHH}=4 \mathrm{~Hz}, 1 \mathrm{H}), 7.11(\mathrm{br},-\mathrm{NH}, 1 \mathrm{H}), 6.75(\mathrm{~d}, \mathrm{JHH}=8 \mathrm{~Hz}$, 1H) $6.69(\mathrm{t}, \mathrm{JHH}=8 \mathrm{~Hz}, 1 \mathrm{H}) 5.75(\mathrm{br},-\mathrm{NH}, 1 \mathrm{H}) \mathrm{ppm} .{ }^{13} \mathrm{C}$ NMR $\left(400 \mathrm{MHz}, \mathrm{DMSO}_{6}\right)$ 163.1, $147.3,141.1,132.8,128.0,127.8,126.8,126.4,116.6,114.5,113.8,66.0$ ppm.

\section{Compound G2.}

${ }^{1} \mathrm{H}$ NMR (400 MHz, DMSO-d 6 , ppm) $\delta: 9.51(\mathrm{~s},-\mathrm{OH}, 1 \mathrm{H}), \delta: 8.09(\mathrm{~s}, 1 \mathrm{H}) \delta: 7.61(\mathrm{~d}, \mathrm{JHH}=$ $4 \mathrm{~Hz}, 1 \mathrm{H}) \delta: 7.31(\mathrm{~d}, \mathrm{JHH}=8 \mathrm{~Hz}, 2 \mathrm{H}) \delta: 7.25(\mathrm{t}, \mathrm{JHH}=8 \mathrm{~Hz}, 1 \mathrm{H}), 6.94(\mathrm{~s}, \mathrm{br},-\mathrm{NH}$, $1 \mathrm{H}), 6.77-6.72(\mathrm{~m}, 3 \mathrm{H}), 6.68(\mathrm{t}, \mathrm{JHH}=8 \mathrm{~Hz}, 1 \mathrm{H}), 5.64(\mathrm{~s}, \mathrm{br},-\mathrm{NH}, 1 \mathrm{H}){ }^{13} \mathrm{C}$ NMR $(400 \mathrm{MHz}$, DMSO-d $\left._{6}\right)$ 164.3, 158.2, 148.7, 133.8, 132.0, 128.8, 127.8, 117.6, 115.5, 114.9, 67.2.

\section{Compound G3}

${ }^{1} \mathrm{H}$ NMR $\left(400 \mathrm{MHz}, \mathrm{DMSO}_{6}\right) \delta: 8.41(\mathrm{~s}, 1 \mathrm{H}), \delta: 7.62(\mathrm{dd}, \mathrm{J} 1=8 \mathrm{~Hz}, \mathrm{~J} 2=8 \mathrm{~Hz}, 1 \mathrm{H}), \delta: 7.53(\mathrm{~d}$, $\left.\mathrm{J}_{\mathrm{HH}}=1.2 \mathrm{~Hz}, 1 \mathrm{H}\right) \delta: 7.44-7.40(\mathrm{~m}, 3 \mathrm{H}) \delta: 7.28-7.22(\mathrm{~m}, 2 \mathrm{H}) \delta: 7.77(\mathrm{~d}, 1 \mathrm{H}) \delta: 6.70-6.66(\mathrm{~m}$, $1 \mathrm{H}), \delta: 5.78(\mathrm{~s}, \mathrm{br},-\mathrm{NH}, 1 \mathrm{H}),{ }^{13} \mathrm{C} \mathrm{NMR}\left(400 \mathrm{MHz}, \mathrm{DMSO} \mathrm{d}_{6}\right) 164.0,148.0,144.9,130.8$, $128.8,127.9,127.3,125.9,117.9,115.4,115.0,66.1$.

\section{Compound G4}

${ }^{1} \mathrm{H} \quad \mathrm{NMR} \quad\left(400 \mathrm{MHz}, \quad \mathrm{DMSO} \quad \mathrm{d}_{6}, \mathrm{ppm}\right) \quad \delta: 8.35(\mathrm{~s}, 1 \mathrm{H}), \delta: 7.62\left(\mathrm{~d}, \mathrm{~J}_{\mathrm{HH}}=8 \mathrm{~Hz}\right.$, 1H) $\delta: 7.53-7.49(\mathrm{~m}, 2 \mathrm{H}), \delta: 7.47-7.44(\mathrm{~m}, 2 \mathrm{H}), \delta: 7.27-7.23(\mathrm{~m}, 1 \mathrm{H}) \delta: 7.15(\mathrm{~s}$, br, $-\mathrm{NH}$, $1 \mathrm{H}), \delta: 6.76\left(\mathrm{~d}, \mathrm{~J}_{\mathrm{HH}}=8 \mathrm{~Hz}, 1 \mathrm{H}\right), \delta: 6.70-6.66(\mathrm{~m}, 1 \mathrm{H}) \delta: 5.77(\mathrm{~s}, \mathrm{br},-\mathrm{NH}, 1 \mathrm{H}),{ }^{13} \mathrm{C}$ NMR $(400$ MHz, DMSO d ${ }_{6}$ ) 164.0, 148.2, 141.2, 133.9, 133.5, 129.3, 128.8, 127.8, 117.8, 115.5, 115.0, 66.3 .

\section{Compound G5}

${ }^{1} \mathrm{H}$ NMR (400 MHz, DMSO-d 6 , ppm) $\delta: 9.41(\mathrm{~s}, 1 \mathrm{H}), \delta: 9.25(\mathrm{~d}, \mathrm{JHH}=8 \mathrm{~Hz}, 2 \mathrm{H}) \delta: 8.93(\mathrm{~d}$, $\mathrm{JHH}=8 \mathrm{~Hz}, 2 \mathrm{H}) \delta: 8.65(\mathrm{~d}, \mathrm{JHH}=8 \mathrm{~Hz}, 1 \mathrm{H}) \delta: 8.44(\mathrm{t}, \mathrm{JHH}=8 \mathrm{~Hz}, 1 \mathrm{H}), \delta: 8.32(\mathrm{t}, \mathrm{JHH}=$ $8 \mathrm{~Hz}, 1 \mathrm{H}), 8.12$ (s, br, -NH, 1H), $7.95(\mathrm{~d}, \mathrm{JHH}=8 \mathrm{~Hz}, 1 \mathrm{H}), 6.97$ (s, br, -NH, 1H) ${ }^{13} \mathrm{C}$ NMR $(400$ MHz, DMSO-d $\mathrm{d}_{6}$ ) 130.6, 122.2, 111.8, 103.3, 96.0, 94.7, 92.7, 91.7, 91.4, 90.7, 89.5, 87.4, 87.3, 81.5 .

\section{Compound G6}

${ }^{1} \mathrm{H} \quad \mathrm{NMR}\left(400 \mathrm{MHz}, \quad \mathrm{DMSO}_{6}, \mathrm{ppm}\right) \delta: 8.20(\mathrm{~s}, 1 \mathrm{H}), \delta: 7.62\left(\mathrm{~d}, \mathrm{~J}_{\mathrm{HH}}=8 \mathrm{~Hz}, 1 \mathrm{H}\right)$ $\delta: 7.44-7.40(\mathrm{~m}, \quad 2 \mathrm{H}), \delta: 7.26-7.22(\mathrm{~m}, 1 \mathrm{H},) \delta: 7.02(\mathrm{~s}$, br, $-\mathrm{NH}, 1 \mathrm{H}),, \delta: 6.92-6.93(\mathrm{~m}$, 2H) $\delta: 6.75\left(\mathrm{~d}, \mathrm{~J}_{\mathrm{HH}}=8 \mathrm{~Hz}, 1 \mathrm{H}\right), \delta: 6.69-6.65(\mathrm{~m}, 1 \mathrm{H}), \delta: 5.71(\mathrm{~s}, \mathrm{br},-\mathrm{NH}, 1 \mathrm{H}), \delta: 3.74(\mathrm{~s}$, $3 \mathrm{H}),{ }^{13} \mathrm{C}$ NMR (400 MHz, DMSO d $\left.\mathrm{d}_{6}\right) 164.2,159.9$, 148.6, 134.0, 133.8, 128.7, 127.9, 117.6, $115.5,114.9,114.1,66.8,55.7$.

\section{Compound G7}

${ }^{1} \mathrm{H}$ NMR $\left(400 \mathrm{MHz}, \mathrm{DMSO} \mathrm{d}_{6}\right) \delta: 8.19(\mathrm{~s}, 1 \mathrm{H}), \delta: 7.61\left(\mathrm{~d}, \mathrm{~J}_{\mathrm{HH}}=8 \mathrm{~Hz}, 1 \mathrm{H}\right) \delta: 7.42\left(\mathrm{~d}, \mathrm{~J}_{\mathrm{HH}}=\right.$ $8 \mathrm{~Hz}, 2 \mathrm{H}) \delta: 7.25\left(\mathrm{t}, \mathrm{J}_{\mathrm{HH}}=4 \mathrm{~Hz}, 1 \mathrm{H}\right) \delta: 7.01(\mathrm{~s}$, br, $-\mathrm{NH}, 1 \mathrm{H}) \delta: 6.96\left(\mathrm{t}, \mathrm{J}_{\mathrm{HH}}=4 \mathrm{~Hz}\right.$, 
2H) $\delta: ~ 6.74-6.75(\mathrm{~m}, 2 \mathrm{H}), \delta: 5.70(\mathrm{~s}, \mathrm{br},-\mathrm{NH}, 1 \mathrm{H}){ }^{13} \mathrm{C}$ NMR $\left(400 \mathrm{MHz}, \mathrm{DMSO} \mathrm{d}_{6}\right) 164.2$, $159.9,148.5,134.0,133.7,128.7,127.9,117.6,115.5,114.9,114.1,79.2,66.8,55.7$.

\section{Compound G8}

${ }^{1} \mathrm{H}$ NMR (400 MHz, DMSO d $\left.\mathrm{d}_{6}\right) \delta: 8.35(\mathrm{~s}, 1 \mathrm{H}), \delta: 7.62-7.59(\mathrm{~m}, 3 \mathrm{H}), \delta: 7.46-7.43(\mathrm{~m}$, $2 \mathrm{H}), \delta: 7.28(\mathrm{~m}, 1 \mathrm{H}), \delta: 7.16(\mathrm{~s}, \mathrm{br},-\mathrm{NH}, 1 \mathrm{H}) \delta: 6.75\left(\mathrm{~d}, \mathrm{~J}_{\mathrm{HH}}=8 \mathrm{~Hz}, 1 \mathrm{H}\right) \delta: 6.70\left(\mathrm{t}, \mathrm{J}_{\mathrm{HH}}=4 \mathrm{~Hz}\right.$, $1 \mathrm{H}), \delta: 5.76(\mathrm{~s}, \mathrm{br},-\mathrm{NH}, 1 \mathrm{H}) .{ }^{13} \mathrm{C}$ NMR (400 MHz, DMSO d 6 ) 164.0, 148.2, 141.6, 133.9, $131.8,129.6,127.9,122.1,117.8,115.5,115.0,66.3$.

\section{Compound G9}

${ }^{1} \mathrm{H}$ NMR (400 MHz, DMSO d $\left.{ }_{6}\right) \delta: 8.18(\mathrm{~s}, 1 \mathrm{H}), \delta: 7.62\left(\mathrm{dd}, \mathrm{J}_{\mathrm{HH}}=1.6 \mathrm{~Hz}, 1 \mathrm{H}\right) \delta: 7.26(\mathrm{~m}$, $1 \mathrm{H}) \delta: 7.13\left(\mathrm{~d}, \mathrm{~J}_{\mathrm{HH}}=2 \mathrm{~Hz}, 1 \mathrm{H}\right) \delta: 7.01-6.93(\mathrm{~m}, 3 \mathrm{H}) \delta: 6.76\left(\mathrm{~d}, \mathrm{~J}_{\mathrm{HH}}=8 \mathrm{~Hz}, 1 \mathrm{H}\right) \delta: 6.69\left(\mathrm{t}, \mathrm{J}_{\mathrm{HH}}=\right.$ $8 \mathrm{~Hz}, 1 \mathrm{H}) \delta: 5.69(\mathrm{~s}, \mathrm{br},-\mathrm{NH}, 1 \mathrm{H}), \delta: 3.75\left(\mathrm{~d}, \mathrm{~J}_{\mathrm{HH}}=4 \mathrm{~Hz}, 6 \mathrm{H}\right){ }^{13} \mathrm{C} \mathrm{NMR}\left(400 \mathrm{MHz}, \mathrm{DMSO} \mathrm{d}_{6}\right)$, 164.3, 149.5, 149.1, 148.6, 134.1, 133.8, 127.9, 119.7, 117.7, 115.6, 115.0, 111.8, 111.1, 67.1, 56.0 .

\section{Compound G10}

${ }^{1} \mathrm{H}$ NMR (400 MHz, DMSO d $\left.{ }_{6}\right) \delta: 8.09(\mathrm{~s}, 1 \mathrm{H}), \delta: 7.61\left(\mathrm{~d}, \mathrm{~J}_{\mathrm{HH}}=8 \mathrm{~Hz}, 1 \mathrm{H}\right) \delta: 7.31\left(\mathrm{~d}, \mathrm{~J}_{\mathrm{HH}}=12\right.$ $\mathrm{Hz}, 2 \mathrm{H}), \delta: 7.31\left(\mathrm{~d}, \mathrm{~J}_{\mathrm{HH}}=12 \mathrm{~Hz}, 2 \mathrm{H}\right) \delta: 7.24\left(\mathrm{t}, \mathrm{J}_{\mathrm{HH}}=8 \mathrm{~Hz}, 1 \mathrm{H}\right), \delta: 6.93(\mathrm{~s},-\mathrm{NH}$, 1H) $\delta: 6.73-6.70(\mathrm{~m}, 3 \mathrm{H}), \delta: 6.68\left(\mathrm{t}, \mathrm{J}_{\mathrm{HH}}=8 \mathrm{~Hz}, 1 \mathrm{H}\right) \delta: 5.63(\mathrm{~s}$, br, $-\mathrm{NH}, 1 \mathrm{H}) \delta: 2.88(\mathrm{~s}$, $6 \mathrm{H}),{ }^{13} \mathrm{C}$ NMR (400 MHz, DMSO d 6 ) 164.3, 151.2, 148.7, 133.7, 129.2, 128.2, 127.9, 117.5, $115.6,114.9,112.5,67.1$.

\section{Compound G11}

${ }^{1} \mathrm{H}$ NMR (400 MHz, DMSO d 6 ) $\delta: 8.27(\mathrm{~s}, 1 \mathrm{H}), \delta: 7.78\left(\mathrm{~d}, \mathrm{~J}_{\mathrm{HH}}=4 \mathrm{~Hz}, 1 \mathrm{H}\right) \delta: 7.68-7.63(\mathrm{~m}$, 2H) $\delta: 7.57-7.54(\mathrm{~m}, 1 \mathrm{H}) \delta: 7.31-7.27(\mathrm{~m}, 1 \mathrm{H}) \delta: 7.07(\mathrm{~s}$, br, $-\mathrm{NH}, 1 \mathrm{H}) \delta: 6.79-6.73(\mathrm{~m}$, 2H) $\delta: 6.07(\mathrm{~s}, \mathrm{br},-\mathrm{NH}, 1 \mathrm{H}),{ }^{13} \mathrm{C}$ NMR $\left(400 \mathrm{MHz}, \mathrm{DMSO} \mathrm{d}_{6}\right)$ 164.0, 148.1, 141.7, 135.4, $134.1,134.0,132.3,127.9,121.9,121.4,118.4,115.2,115.2,66.7$.

\section{Compound G12}

${ }^{1} \mathrm{H}$ NMR (400 MHz, DMSO d $\left.\mathrm{d}_{6}\right) \delta: 8.55(\mathrm{~s}, \mathrm{br},-\mathrm{NH}, 1 \mathrm{H}), \delta: 7.98\left(\mathrm{t}, \mathrm{J}_{\mathrm{HH}}=16 \mathrm{~Hz}\right.$, 1H) $\delta: 7.85-7.61(\mathrm{~m}, 3 \mathrm{H}) \delta: 7.58-7.48(\mathrm{~m}, 5 \mathrm{H}) \delta: 7.26\left(\mathrm{t}, \mathrm{J}_{\mathrm{HH}}=16 \mathrm{~Hz}, 1 \mathrm{H}\right) \delta: 7.10(\mathrm{~s}$, 1H) $\delta: 6.77-6.69(\mathrm{~m}, 1 \mathrm{H}) \delta: 6.48(\mathrm{~s}$, br, $-\mathrm{NH}, 1 \mathrm{H}),{ }^{13} \mathrm{C}$ NMR $\left(400 \mathrm{MHz}\right.$, DMSO $\left.\mathrm{d}_{6}\right)$ $154.1,136.65,134.31,133.8,133.6,131.0,129.8,129.1,127.5,125.7,125.7,125.1,117.7,66.4$.

\section{Compound G13}

${ }^{1} \mathrm{H}$ NMR (400 MHz, DMSO d 6 ) $\delta: 8.74(\mathrm{~s}, 1 \mathrm{H}), \delta: 8.38(\mathrm{~s}, 1 \mathrm{H}) \delta: 8.24-8.14(\mathrm{~m}, 2 \mathrm{H}) \delta: 7.79-$ $7.77(\mathrm{~m}, \quad 2 \mathrm{H}) \delta: 7.56-7.47(\mathrm{~m}, \quad 6 \mathrm{H}) \delta: 1.23(\mathrm{~s}, \quad 1 \mathrm{H}), \delta: 7.32-7.28(\mathrm{~m}, \quad 1 \mathrm{H}),) \delta: 7.16(\mathrm{~s}$, $1 \mathrm{H}), \delta: 6.79 .32-6.72(\mathrm{~m}, 1 \mathrm{H}),{ }^{13} \mathrm{C} \mathrm{NMR}(400 \mathrm{MHz}$, DMSO d 6 ) 162.4, 149.3, 135.7, 135.1, $134.3,133.6,132.2$, 131.0, 129.9, 129.1, 128.1, 127.6, 126.9, 126.6, 126.3, 125.7, 125.6, 125.1, $117.8,115.1,66.4$.

\section{Compound G14}

${ }^{1} \mathrm{H}$ NMR $\left(400 \mathrm{MHz}, \mathrm{DMSO} \mathrm{d}_{6}\right) \delta: 8.84(\mathrm{~s}, 1 \mathrm{H}), \delta: 8.17\left(\mathrm{~d}, \mathrm{~J}_{\mathrm{HH}}=8 \mathrm{~Hz}, 2 \mathrm{H}\right) \delta: 7.94\left(\mathrm{t}, \mathrm{J}_{\mathrm{HH}}=8 \mathrm{~Hz}\right.$, $2 \mathrm{H}) \delta: 7.84\left(\mathrm{t}, \mathrm{J}_{\mathrm{HH}}=8 \mathrm{~Hz}, 2 \mathrm{H}\right) \delta: 7.60-7.55(\mathrm{~m}, 2 \mathrm{H}) \delta: 7.40\left(\mathrm{t}, \mathrm{J}_{\mathrm{HH}}=8 \mathrm{~Hz}, 1 \mathrm{H}\right), \delta: 7.21\left(\mathrm{~d}, \mathrm{~J}_{\mathrm{HH}}=\right.$ 
$8 \mathrm{~Hz}, 1 \mathrm{H}),{ }^{13} \mathrm{C}$ NMR (400 MHz, DMSO d 6 ) 168.1, 158.8, 149.7, 149.3, 134.5, 132.9, 132.3, 130.3, 130.0, 129.8, 127.1, 125.2, 119.7 .
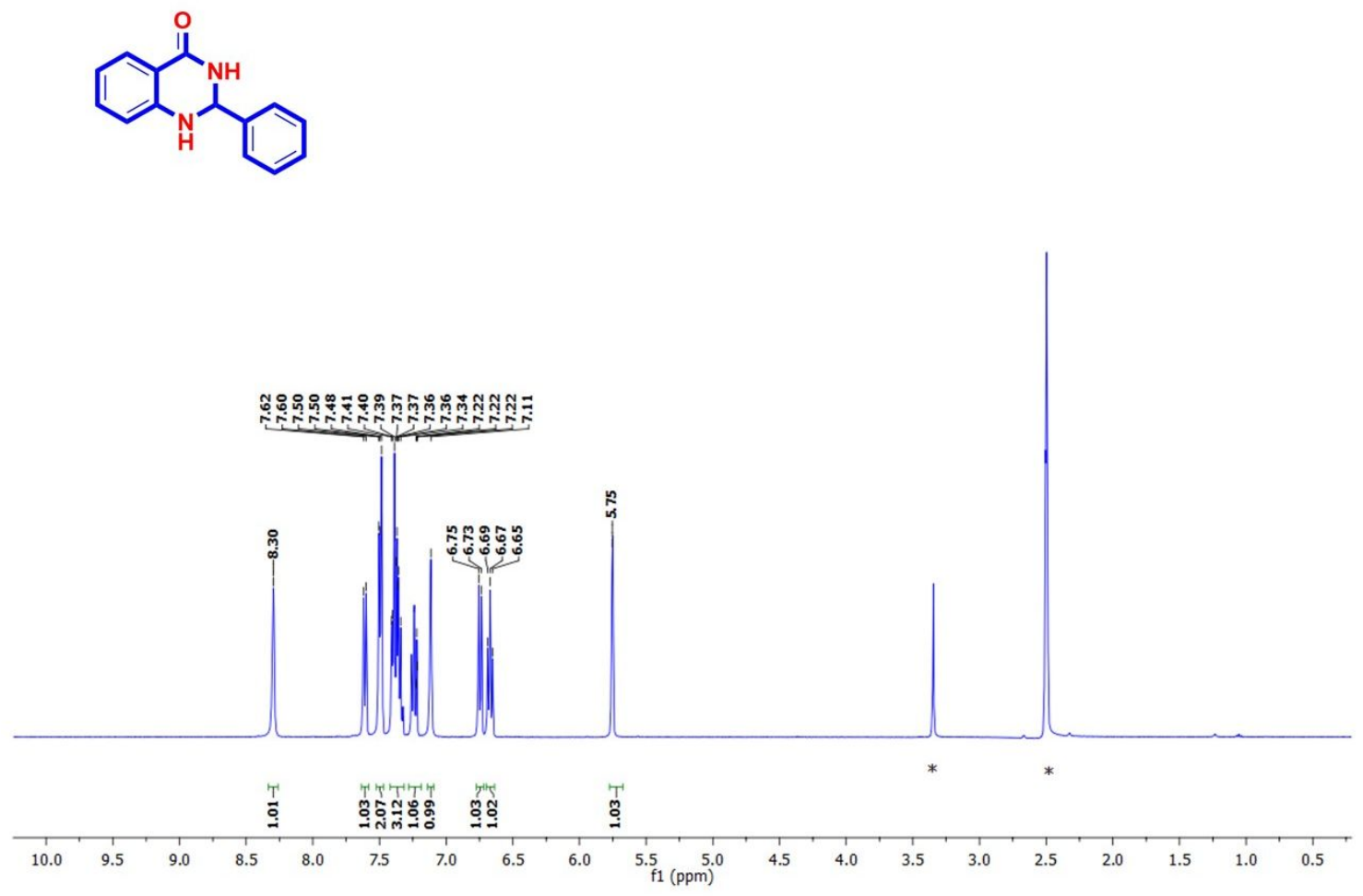

Compound G1 ${ }^{1} \mathrm{H}$ NMR

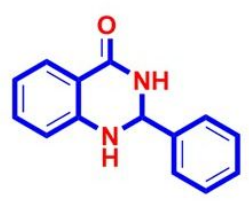

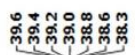

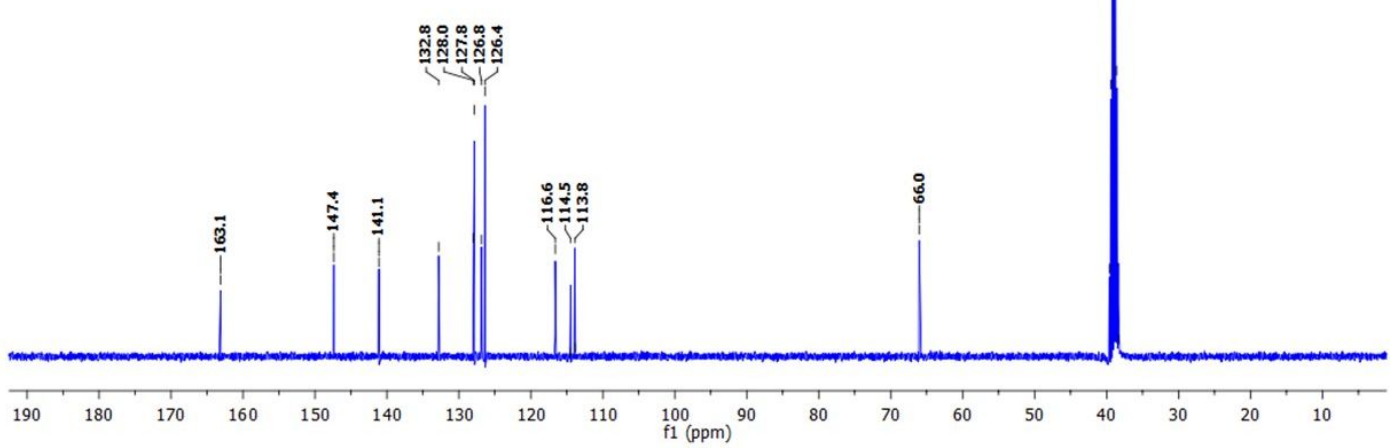

Compound G1 ${ }^{13} \mathrm{C}$ NMR

S-9 

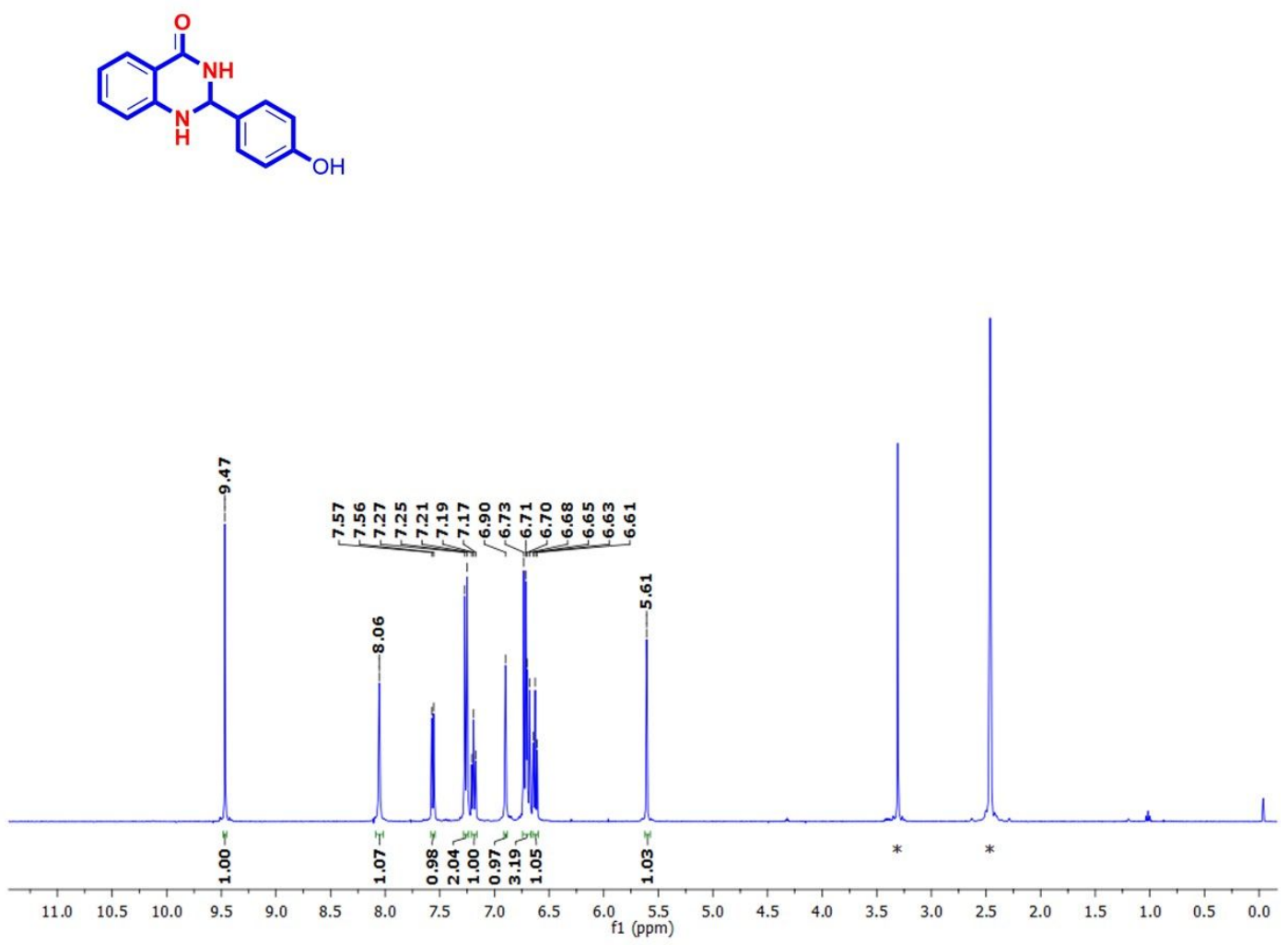

Compound G2 ${ }^{1} \mathrm{H}$ NMR
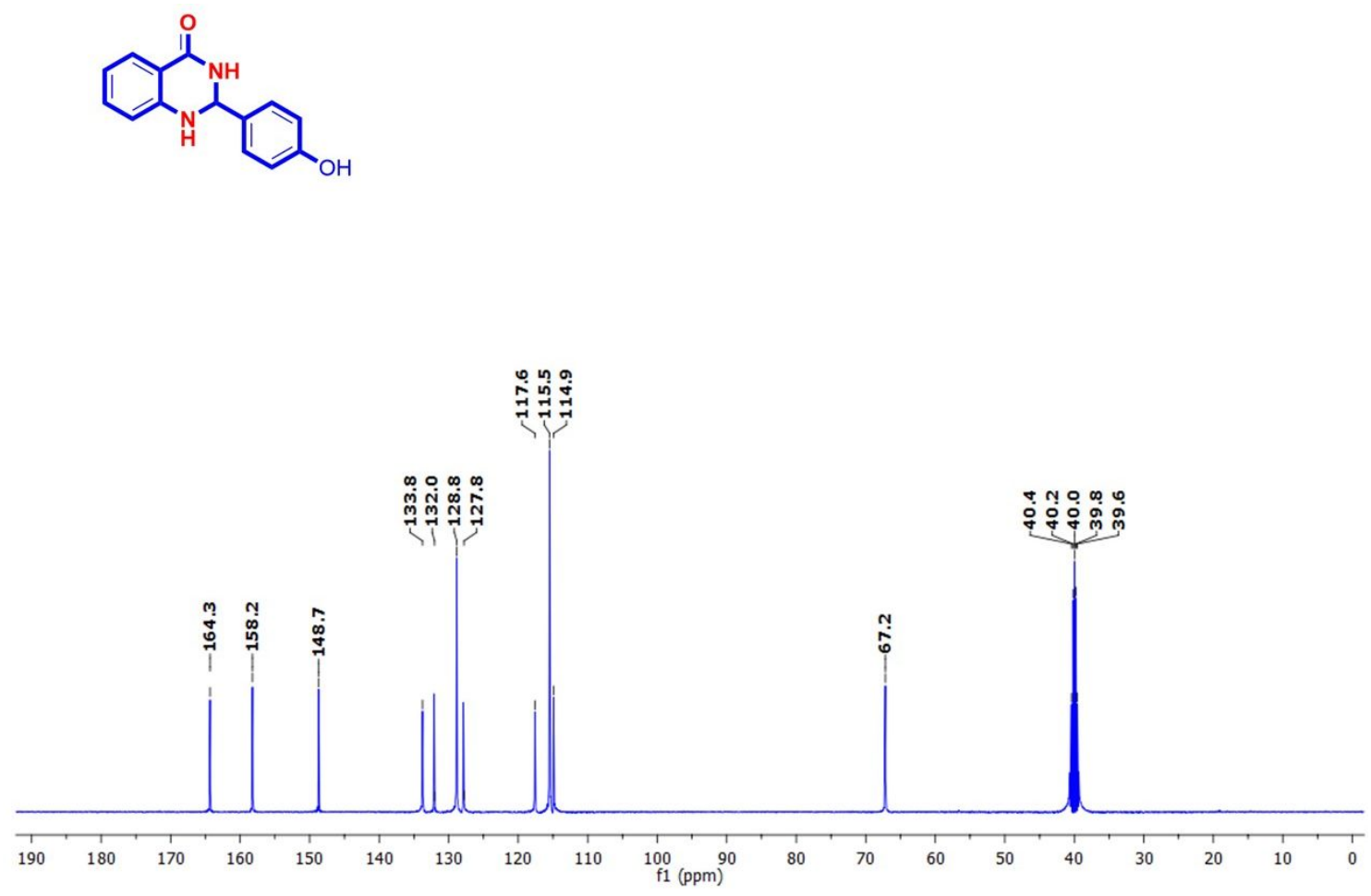

Compound G2 ${ }^{13} \mathrm{C}$ NMR

S-10 
<smiles>[GeH2]</smiles>

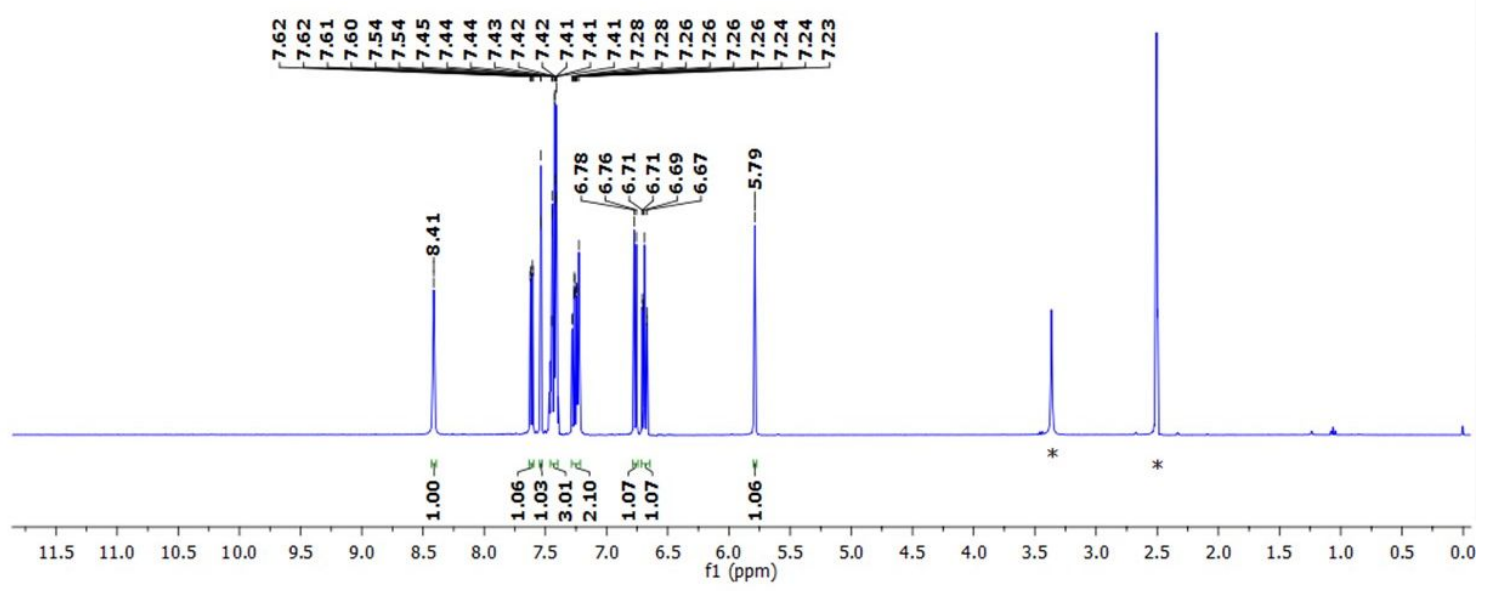

Compound G3 ${ }^{1} \mathrm{H}$ NMR<smiles>O=C1NC(c2cccc(Br)c2)Nc2ccccc21</smiles>

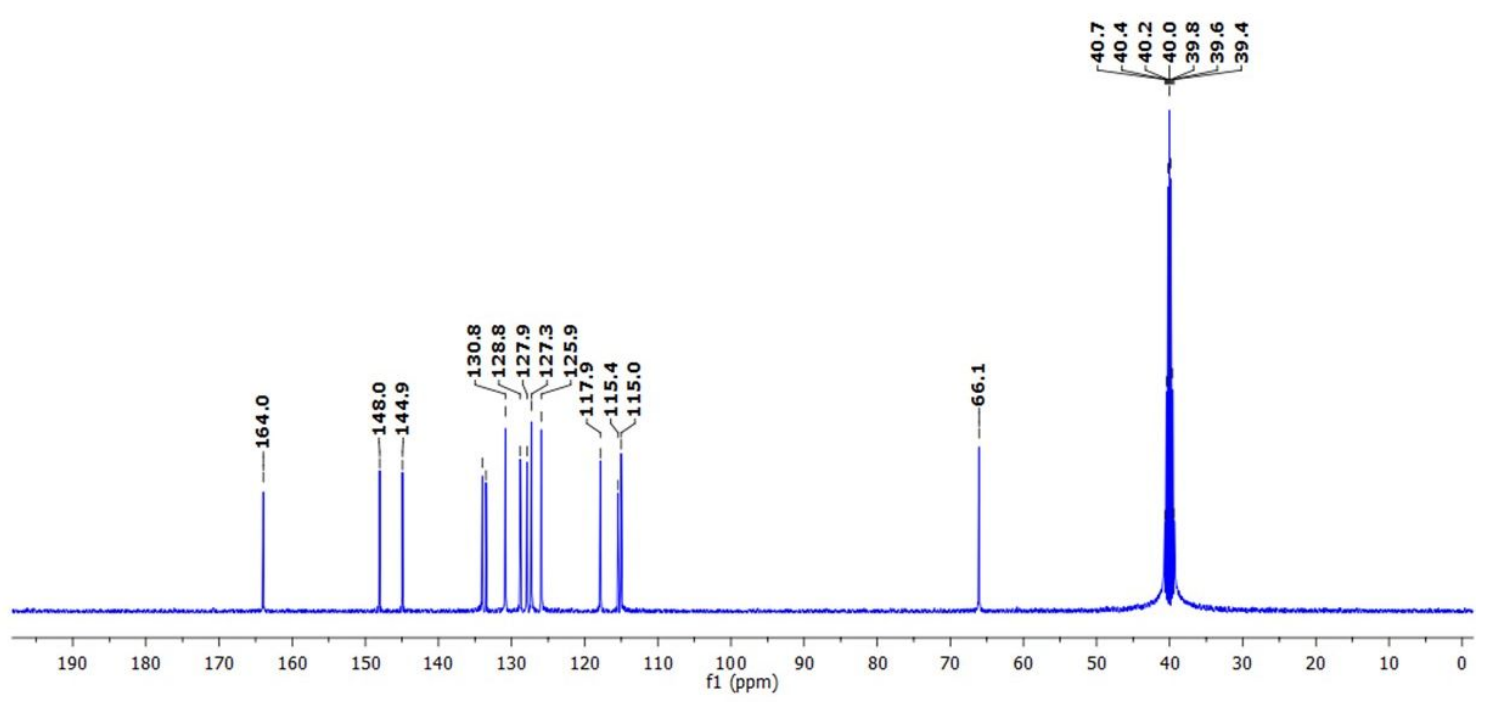

Compound G3 ${ }^{13} \mathrm{C}$ NMR 
<smiles>O=C1NC(c2ccc(Cl)cc2)Nc2ccccc21</smiles>
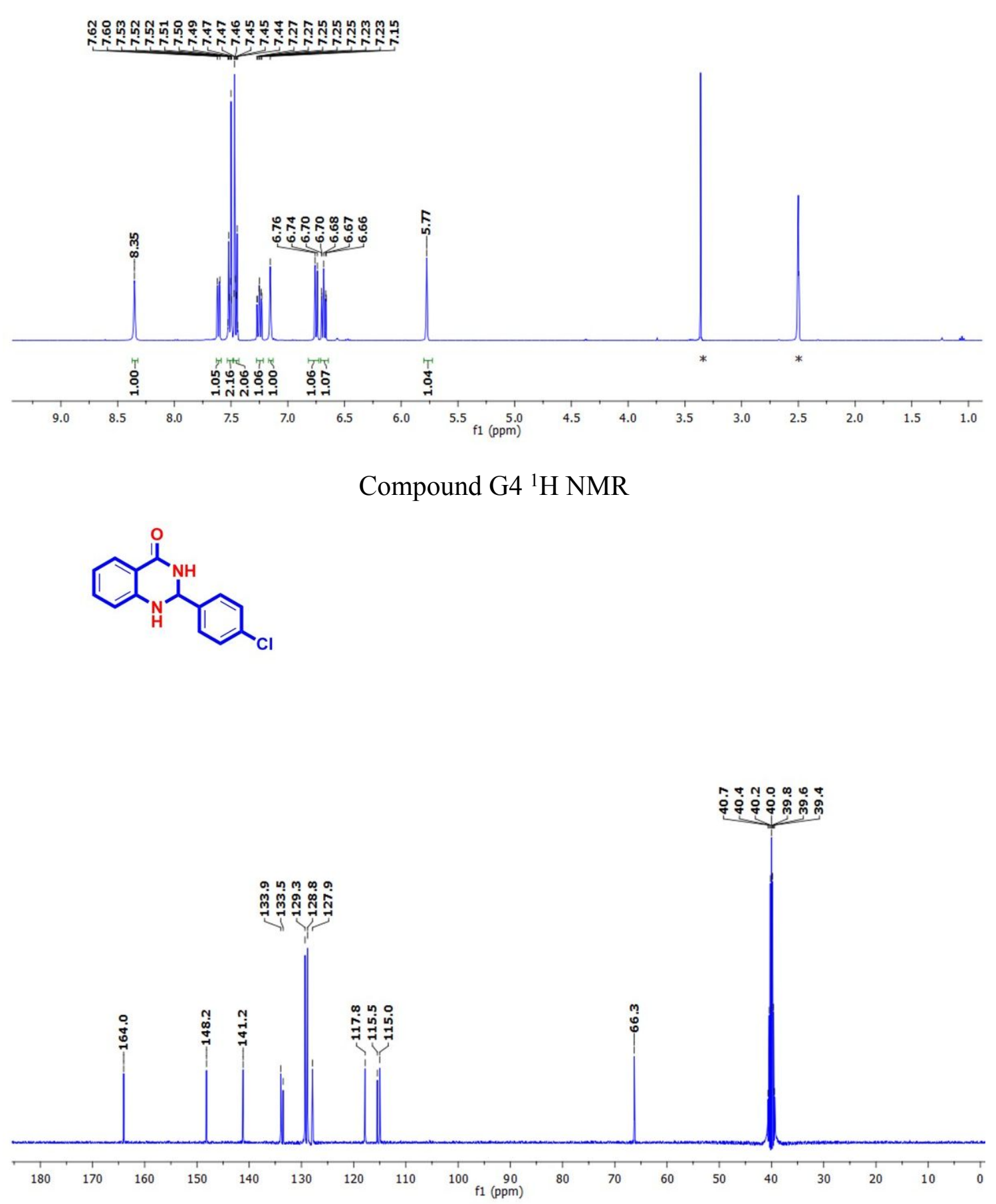

Compound G4 ${ }^{13} \mathrm{C}$ NMR

S-12 
<smiles>O=C1NC(c2ccc([N+](=O)[O-])cc2)Nc2ccccc21</smiles>

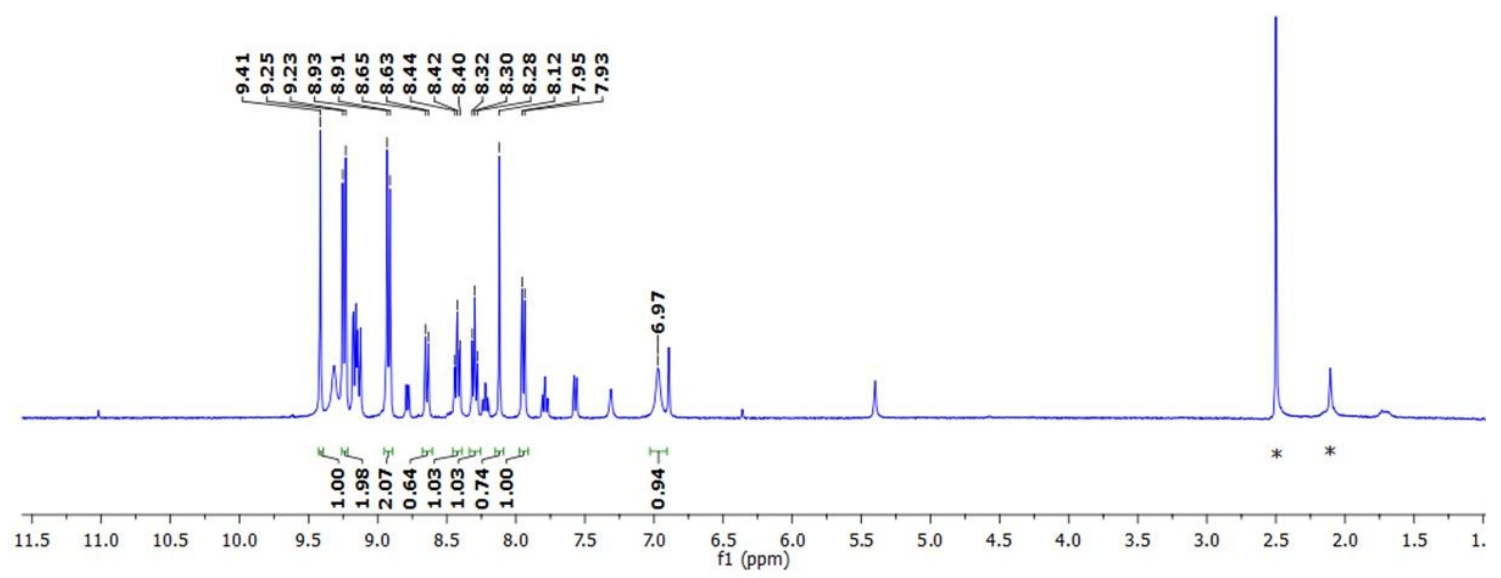

Compound G5 ${ }^{1} \mathrm{H}$ NMR
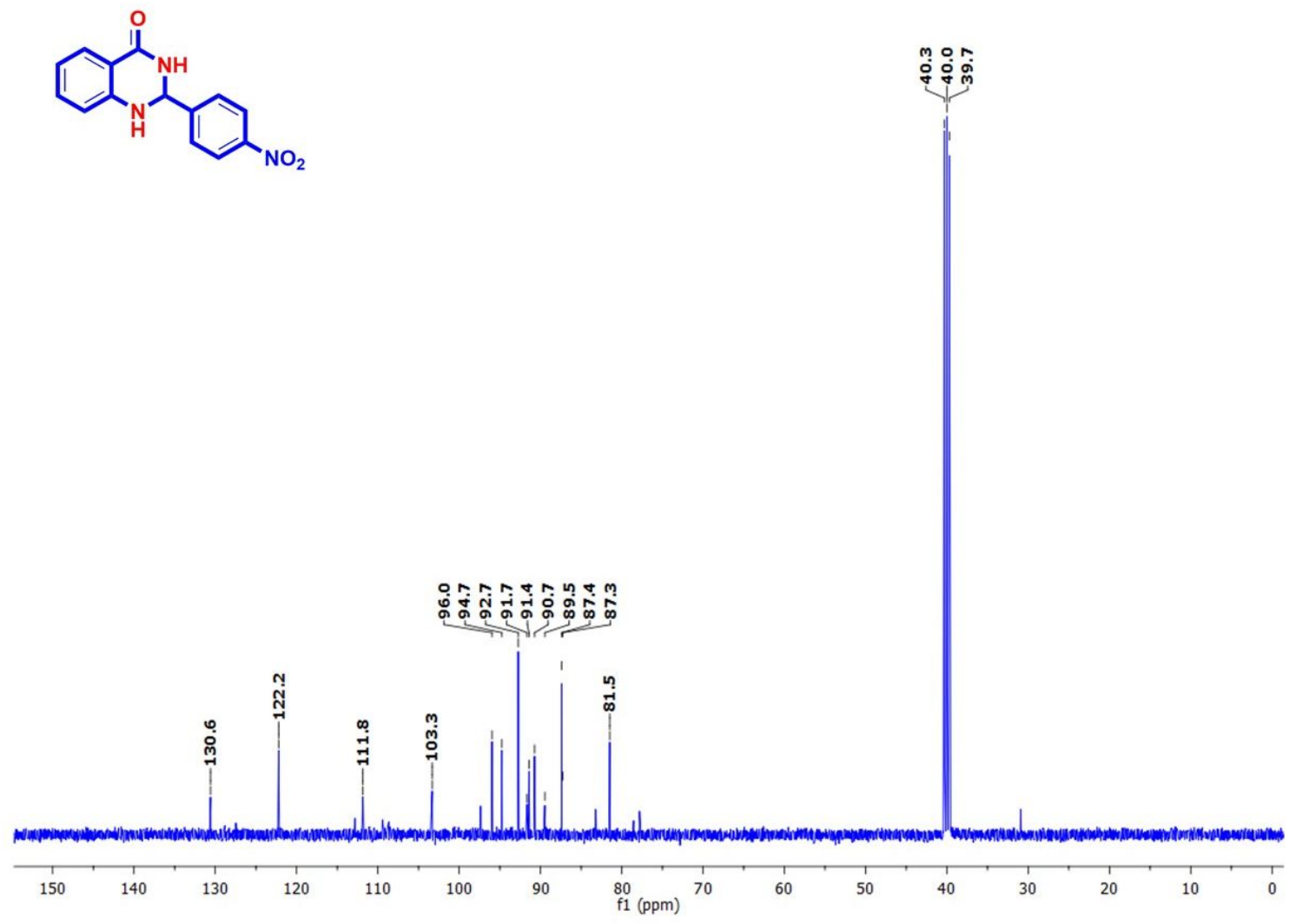

Compound G5 ${ }^{13} \mathrm{C}$ NMR

S-13 


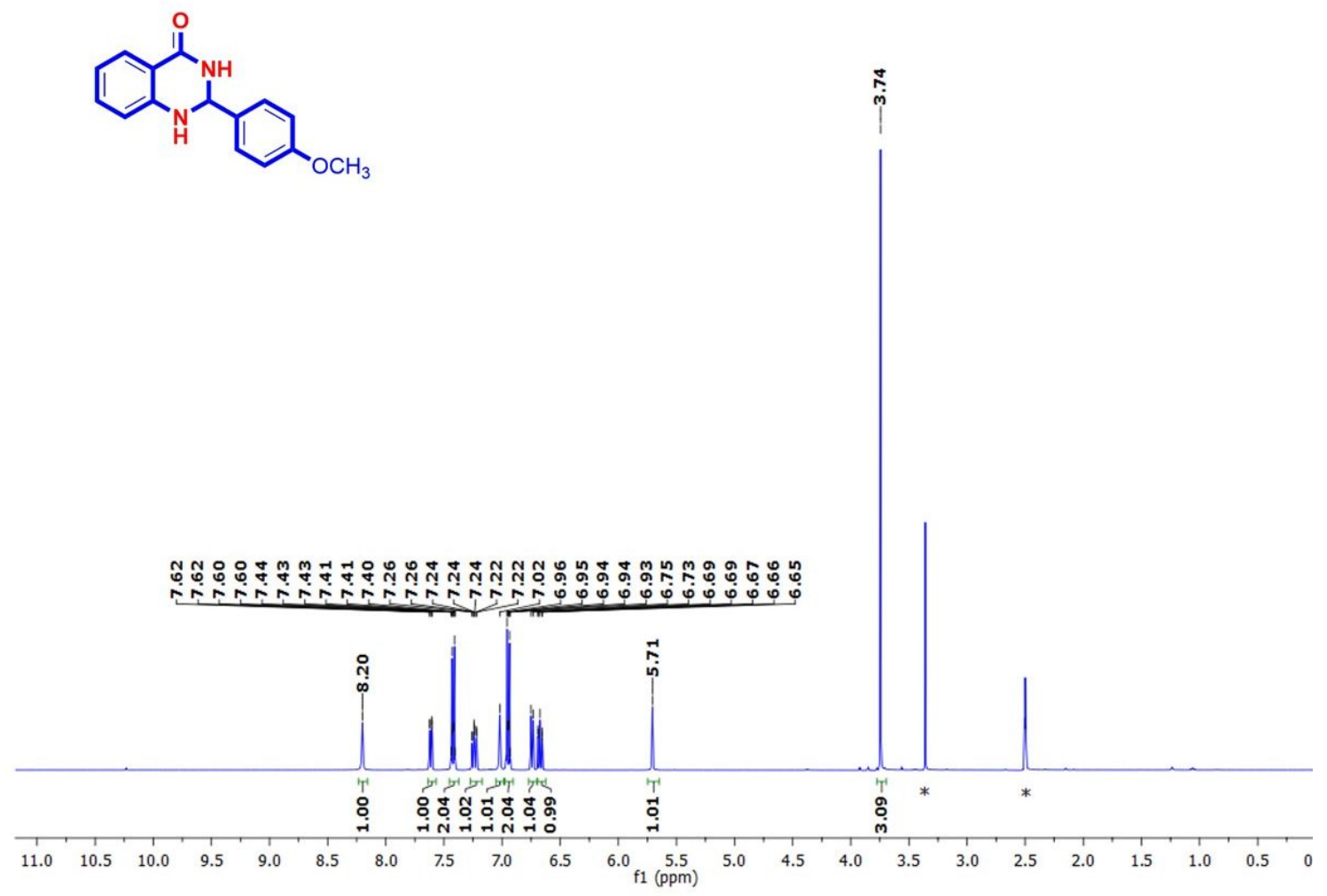

Compound G6 ${ }^{1} \mathrm{H}$ NMR
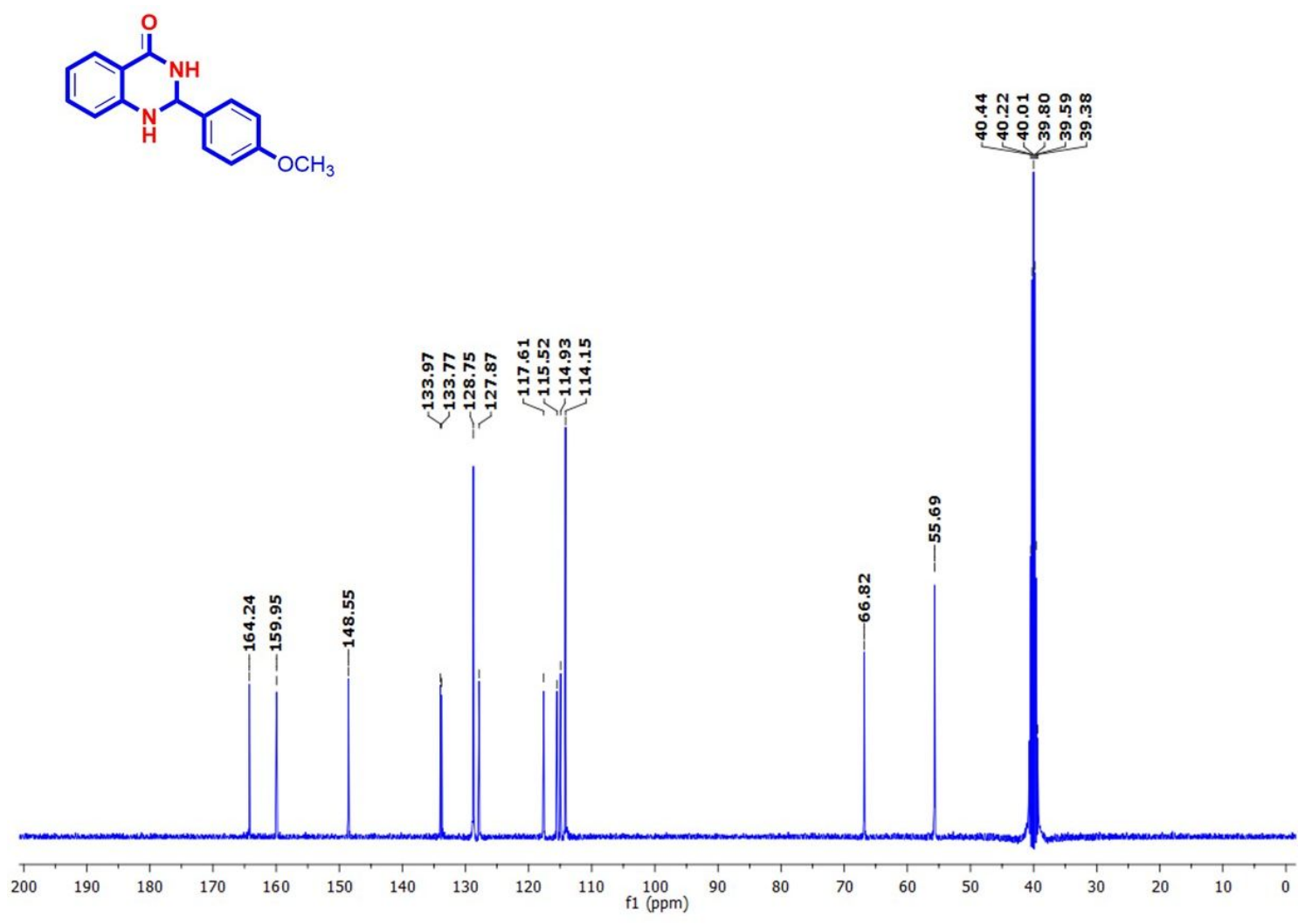

Compound G6 ${ }^{13} \mathrm{C}$ NMR 

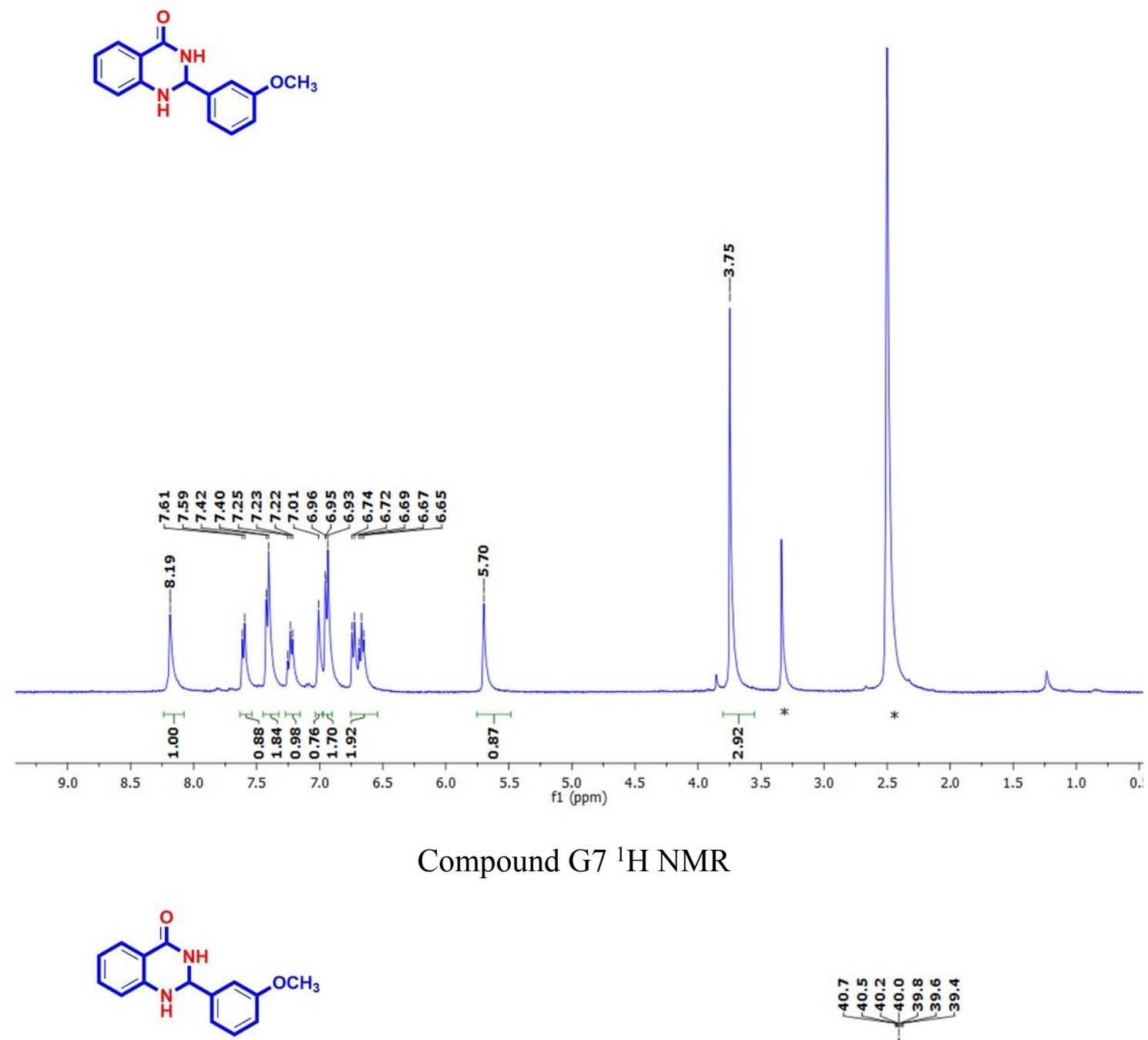

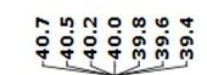

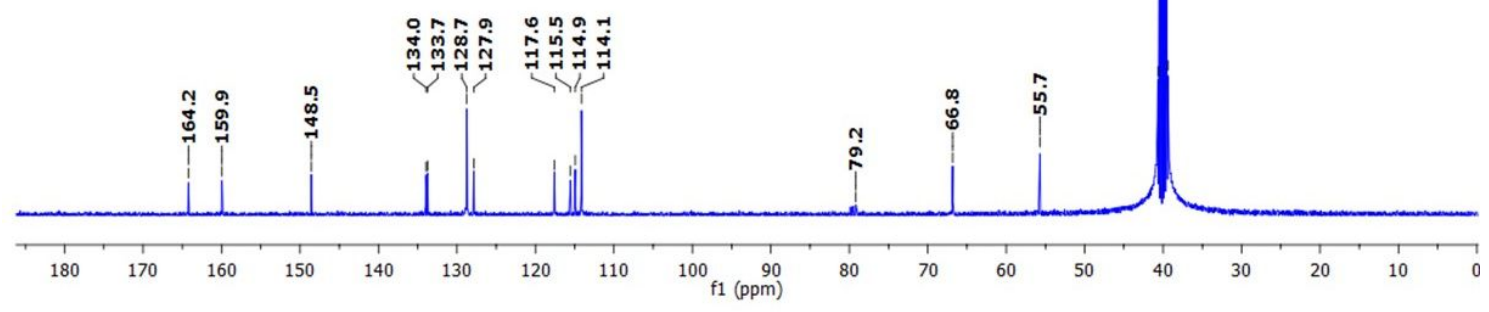

Compound G7 ${ }^{13} \mathrm{C}$ NMR

S-15 

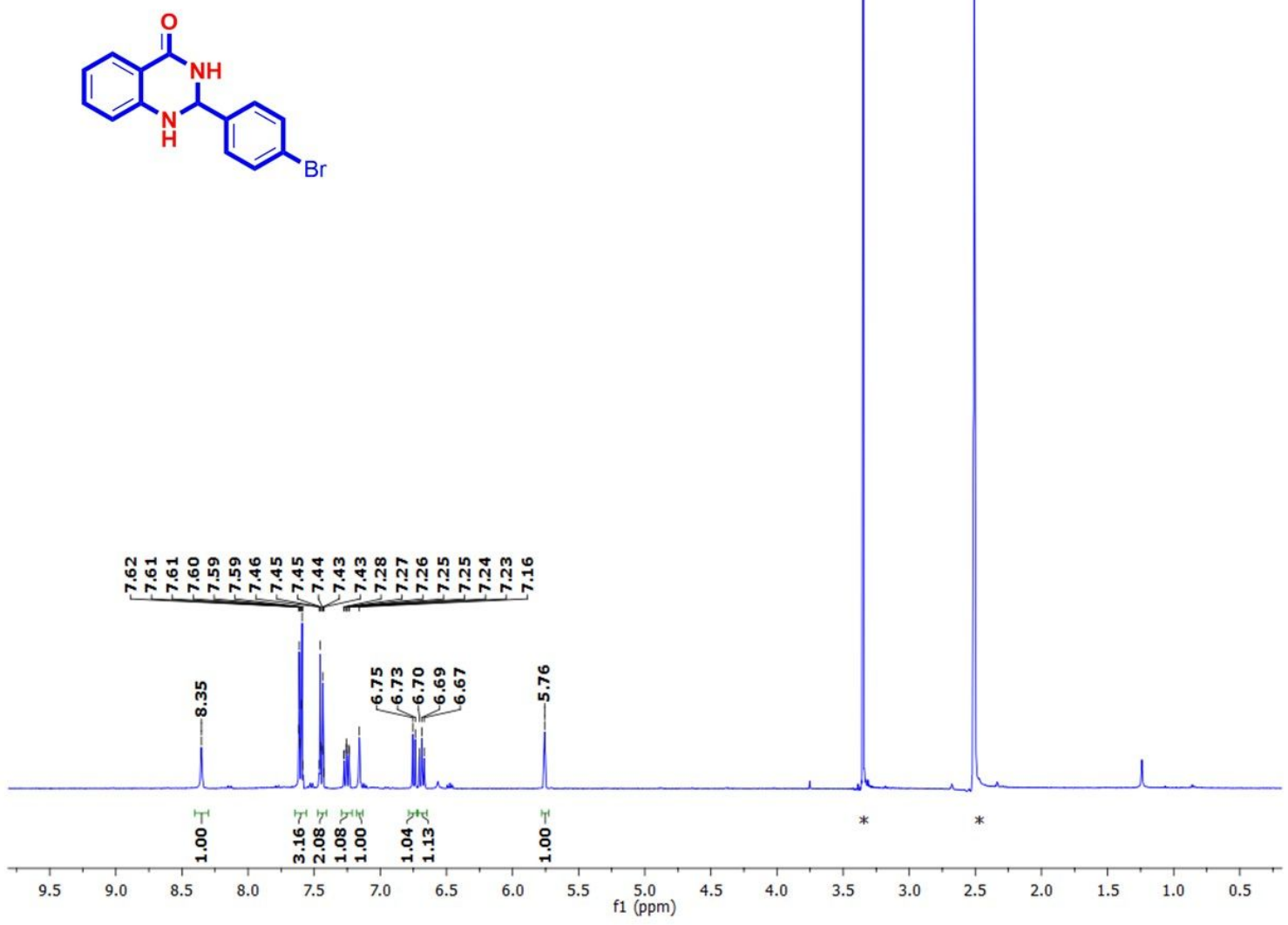

Compound G8 ${ }^{1} \mathrm{H}$ NMR
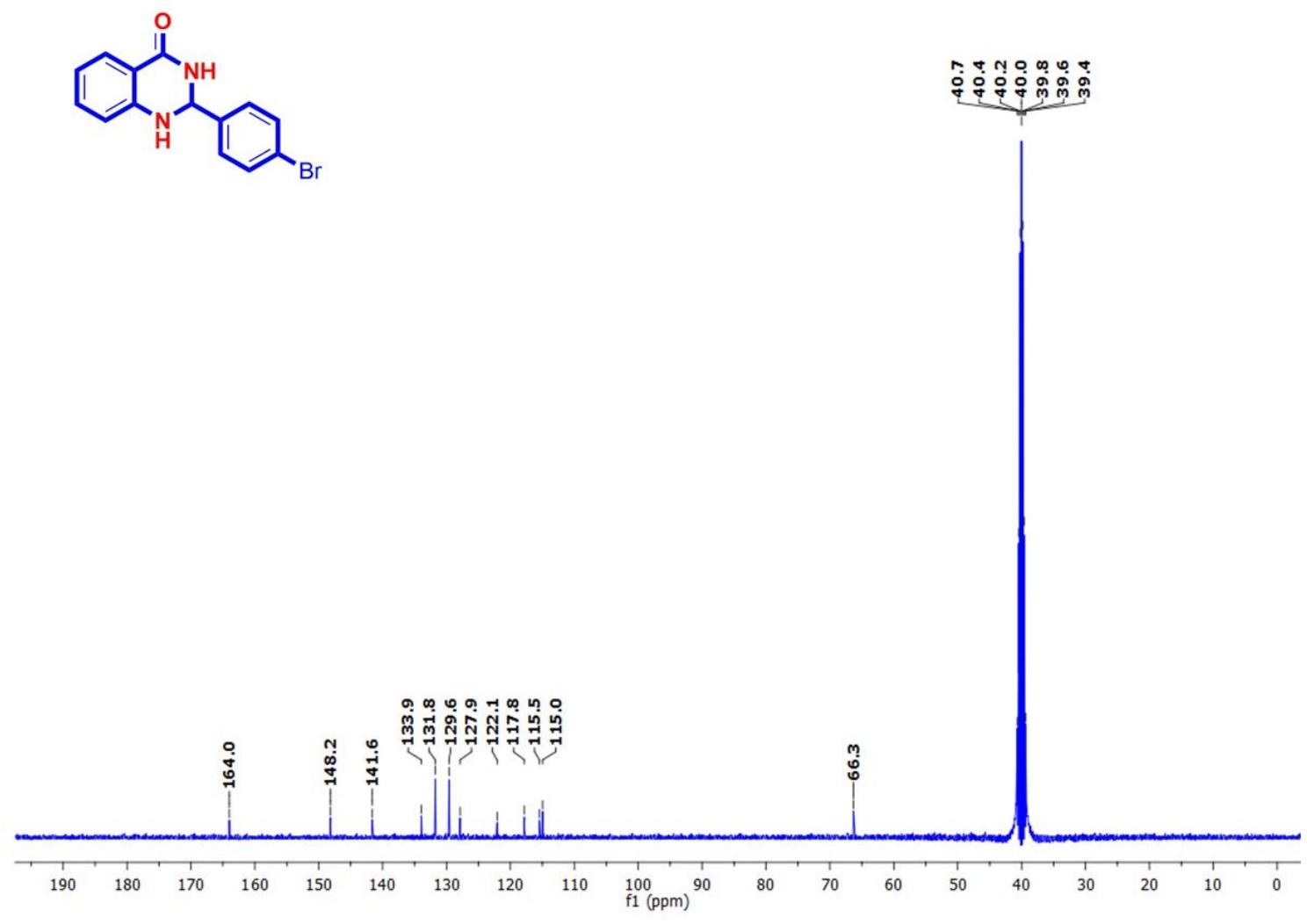

Compound G8 ${ }^{13} \mathrm{C}$ NMR

S-16 


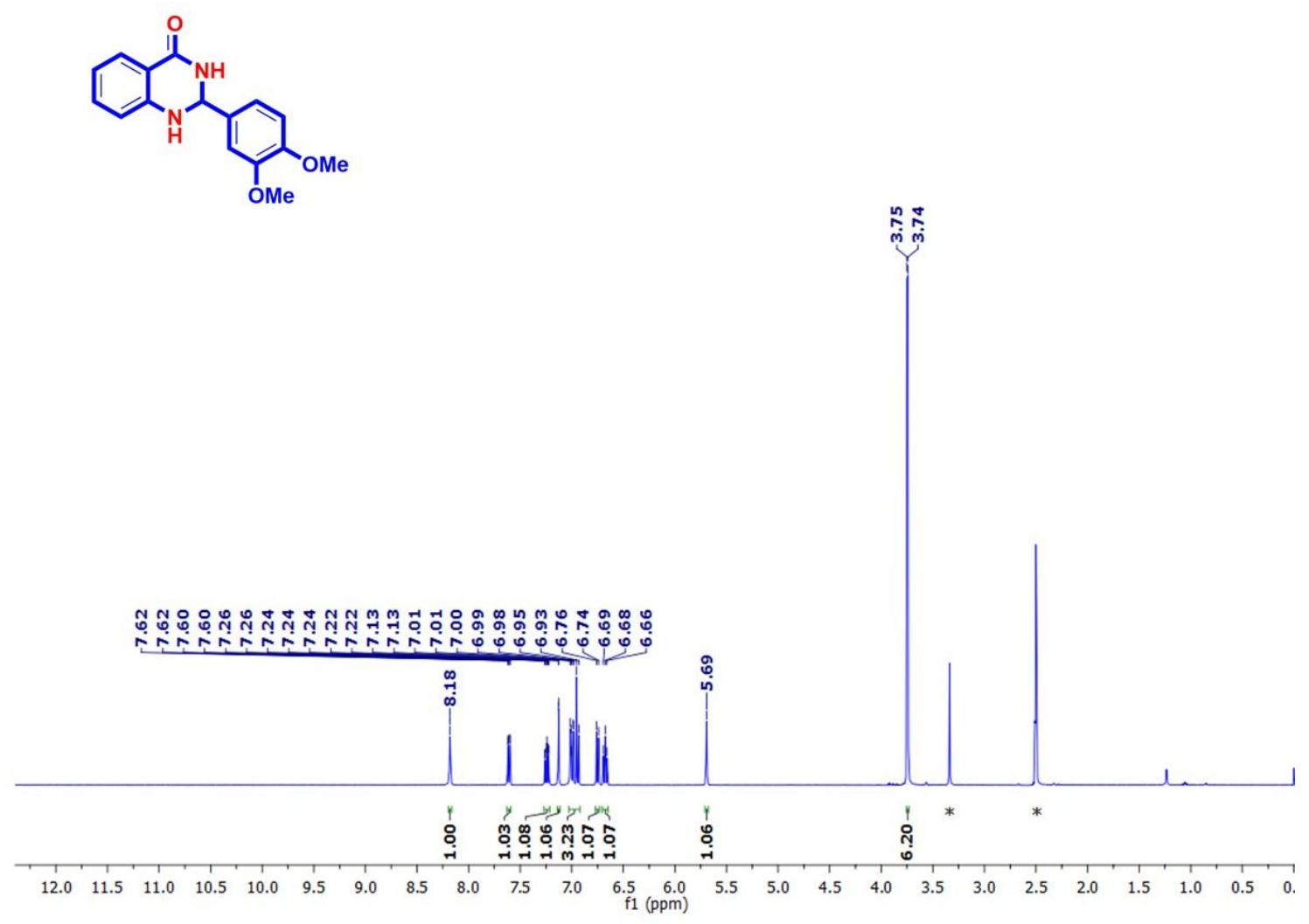

Compound G9 ${ }^{1} \mathrm{H}$ NMR

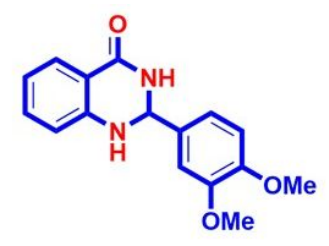

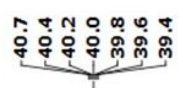

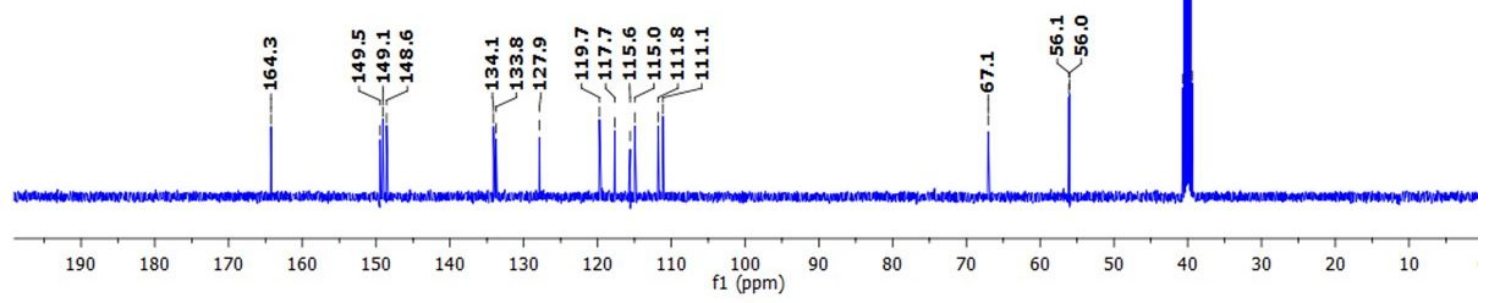

Compound G9 ${ }^{13} \mathrm{C}$ NMR 

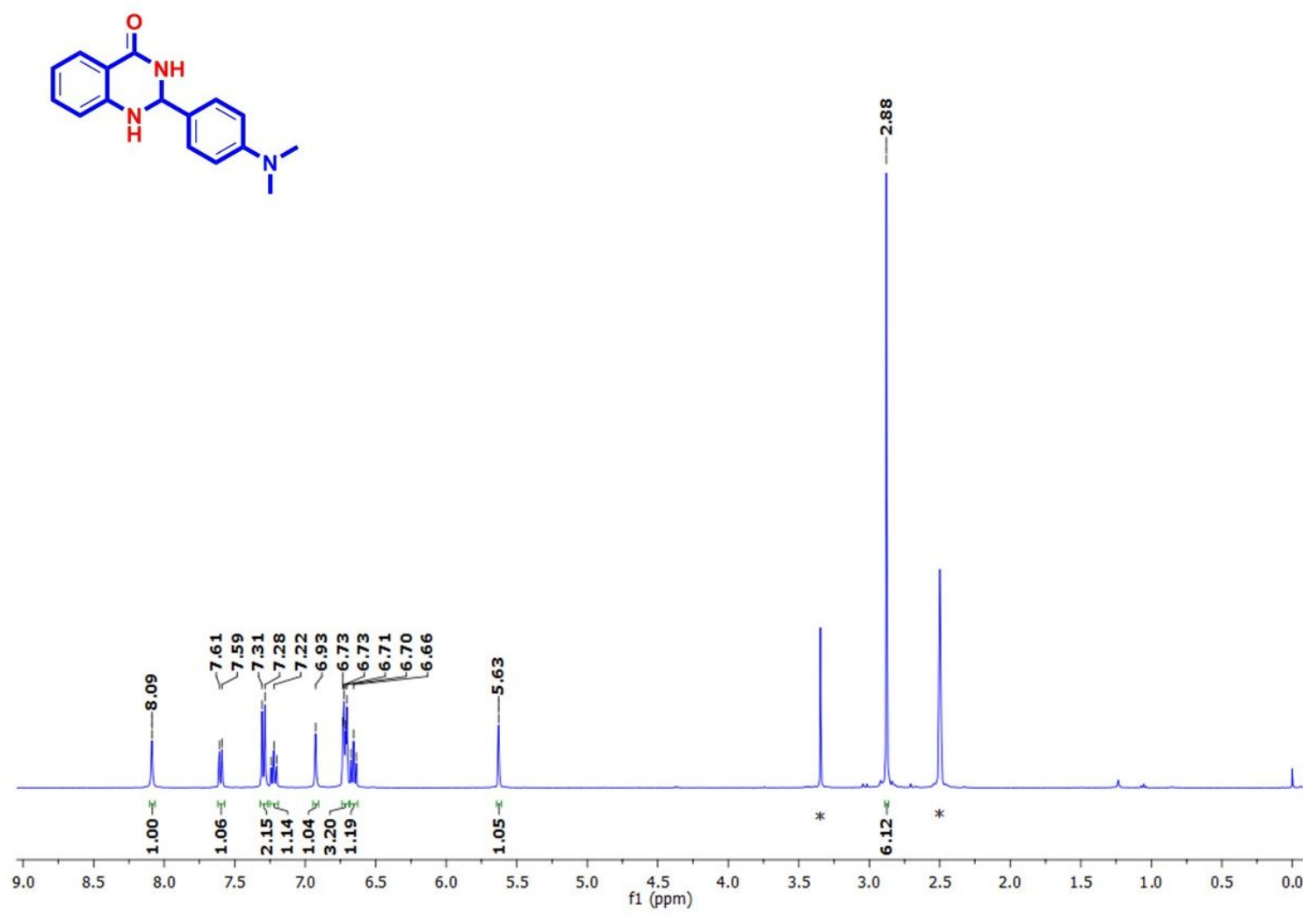

Compound G10 ${ }^{1} \mathrm{H}$ NMR

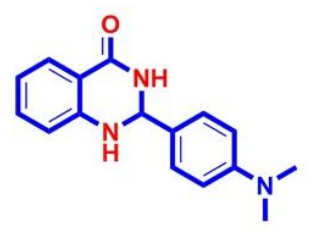

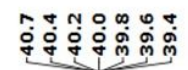

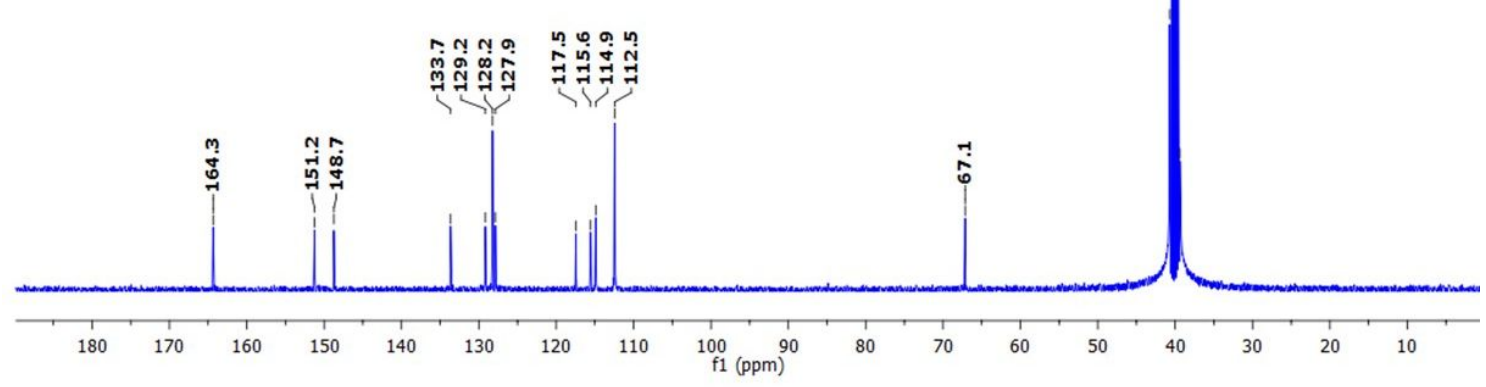

Compound G10 ${ }^{13} \mathrm{C}$ NMR

S-18 

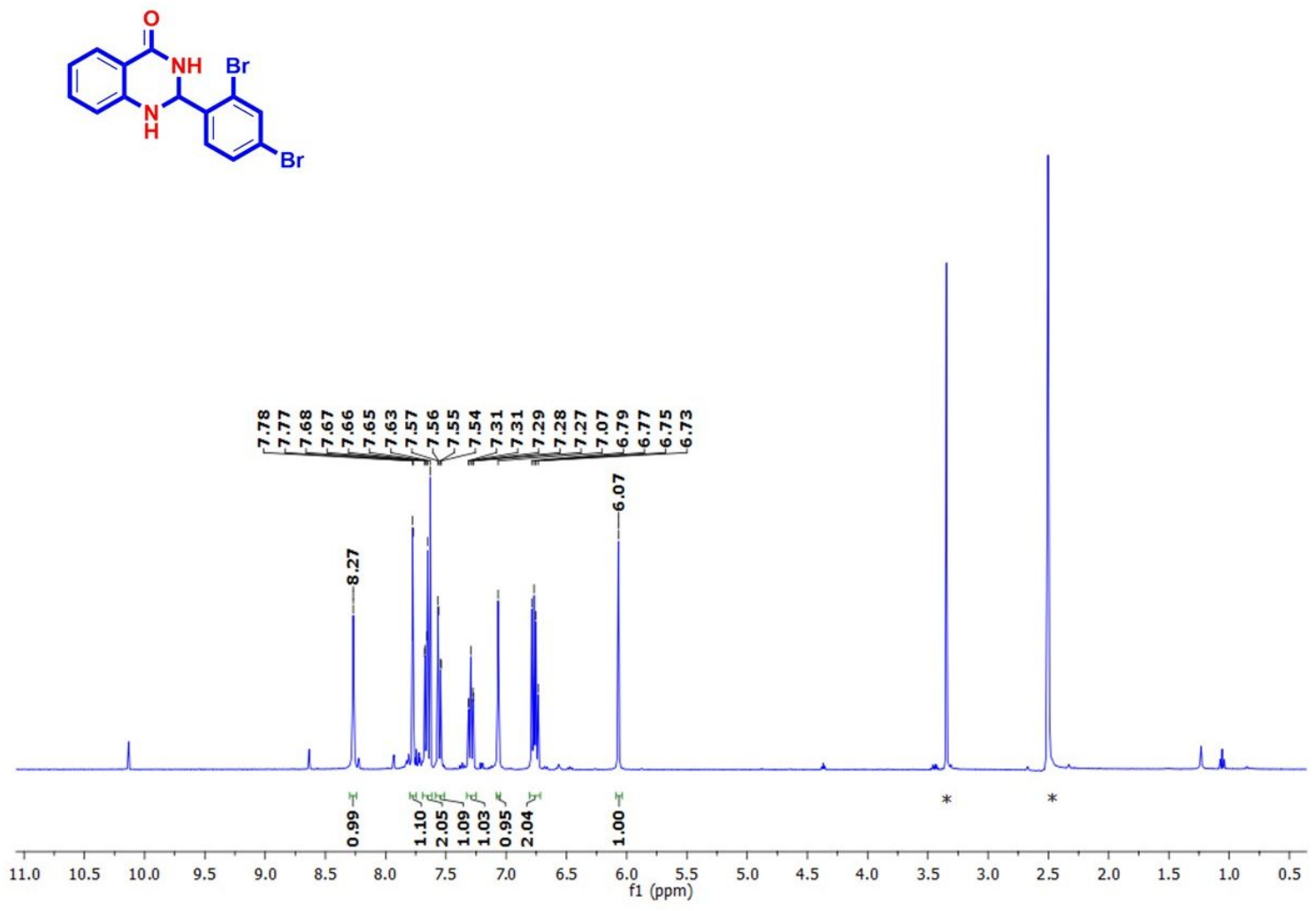

Compound G1 $1{ }^{1} \mathrm{H}$ NMR
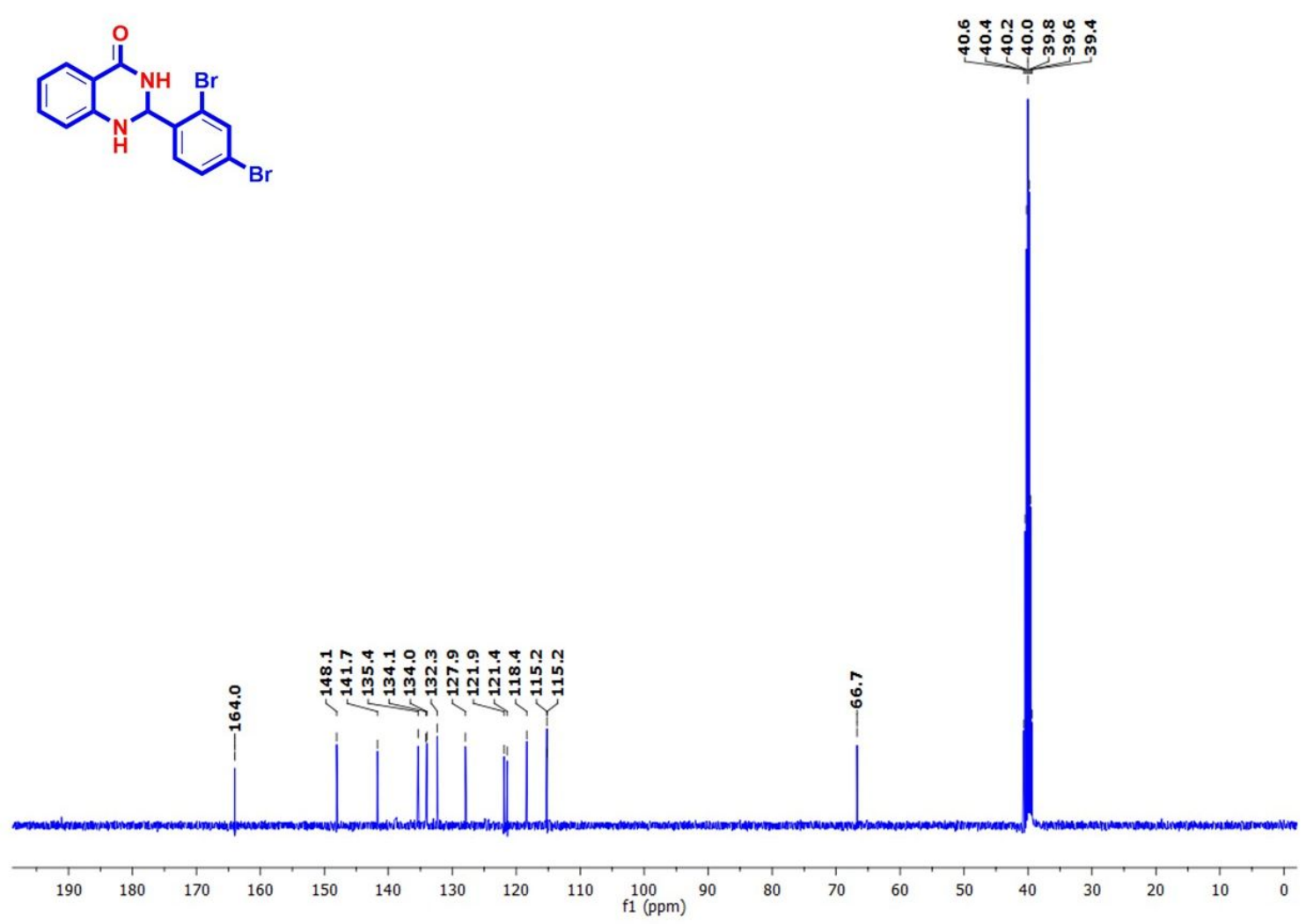

Compound G1 $1{ }^{13} \mathrm{C}$ NMR 


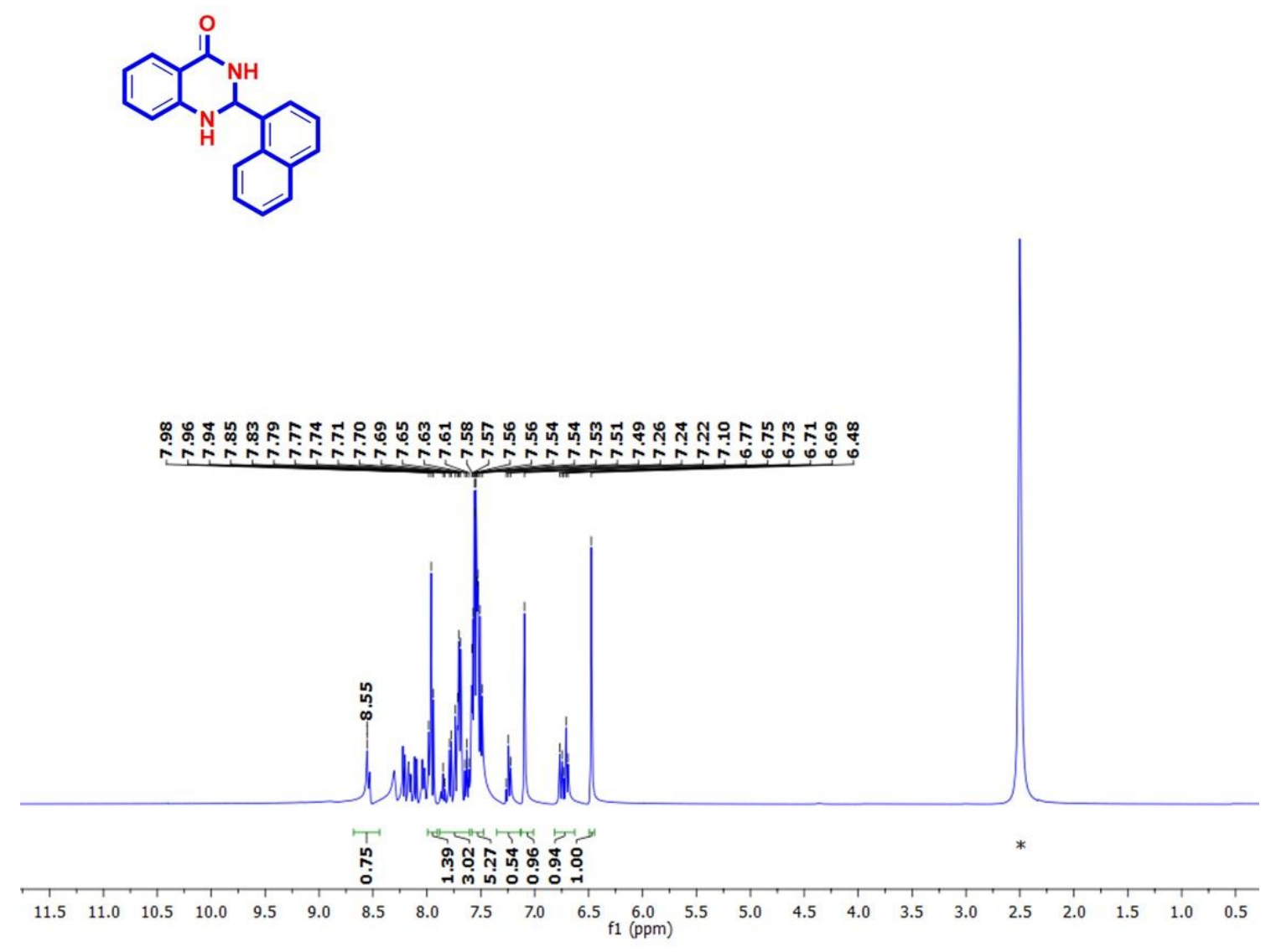

Compound G12 ${ }^{1} \mathrm{H}$ NMR 


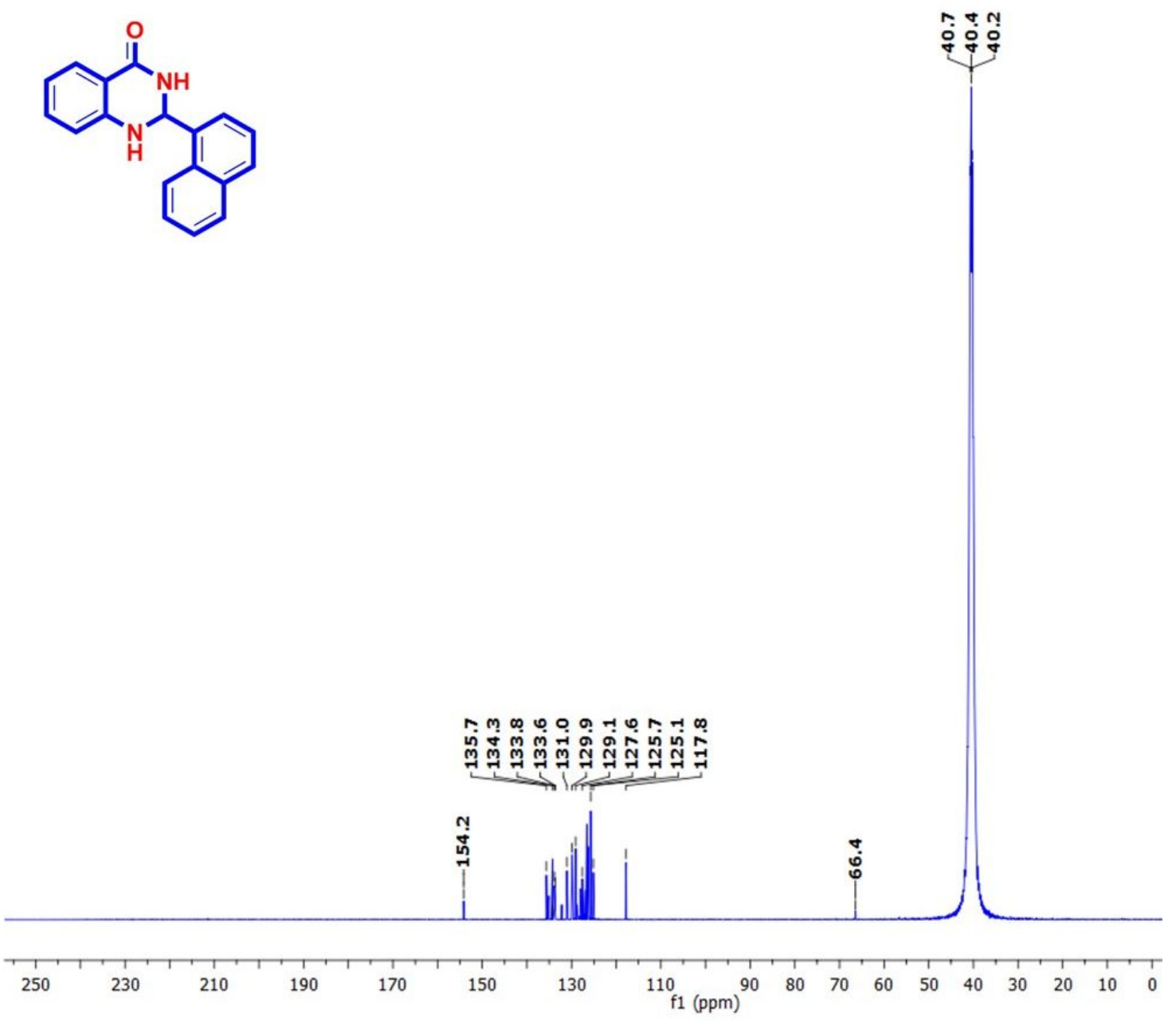

Compound G12 ${ }^{13} \mathrm{C}$ NMR 


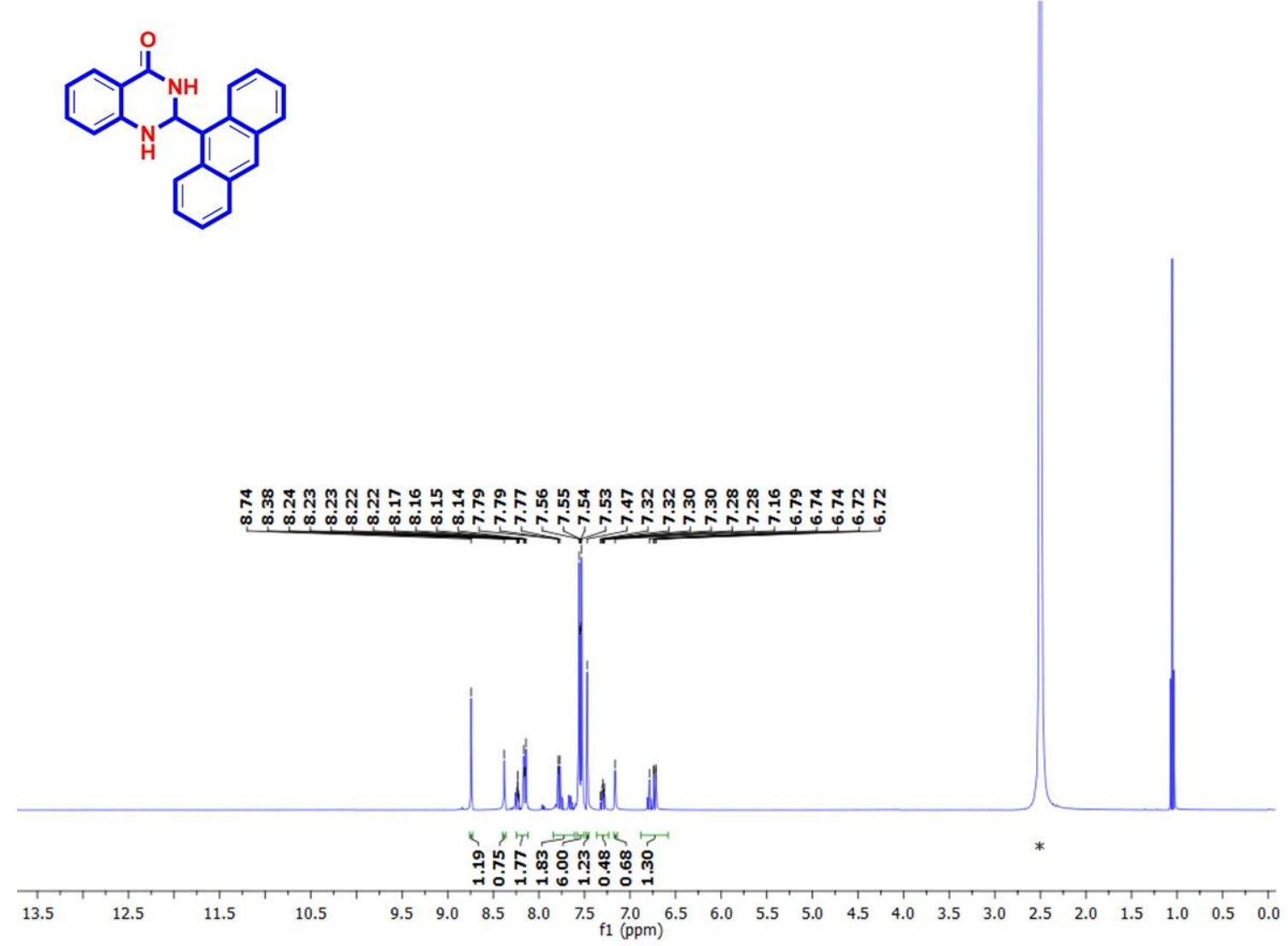

Compound G13 ${ }^{1} \mathrm{H}$ NMR<smiles>O=C1NC(c2c3ccccc3cc3ccccc23)Nc2ccccc21</smiles>

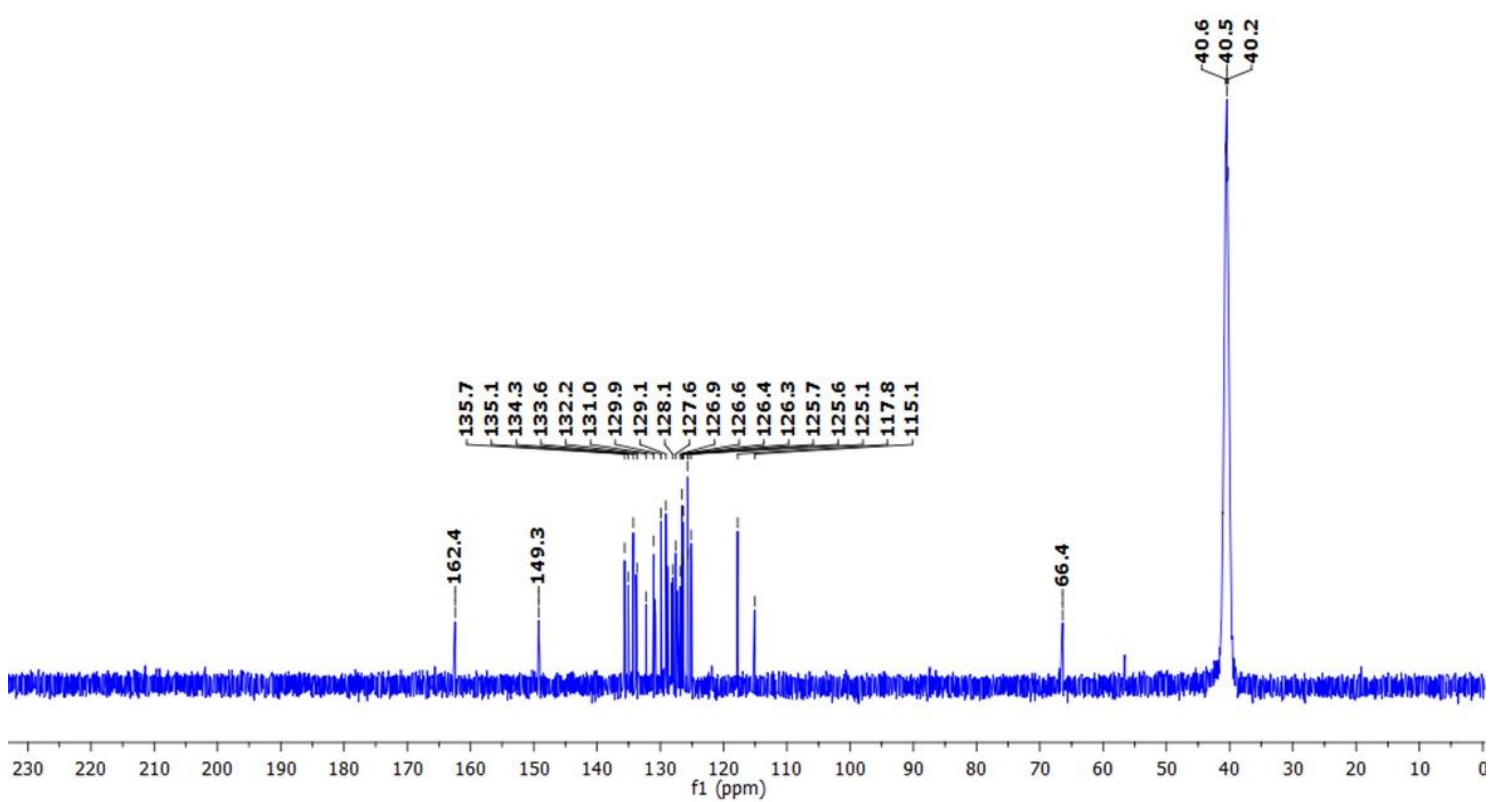

Compound G13 ${ }^{13} \mathrm{C}$ NMR 


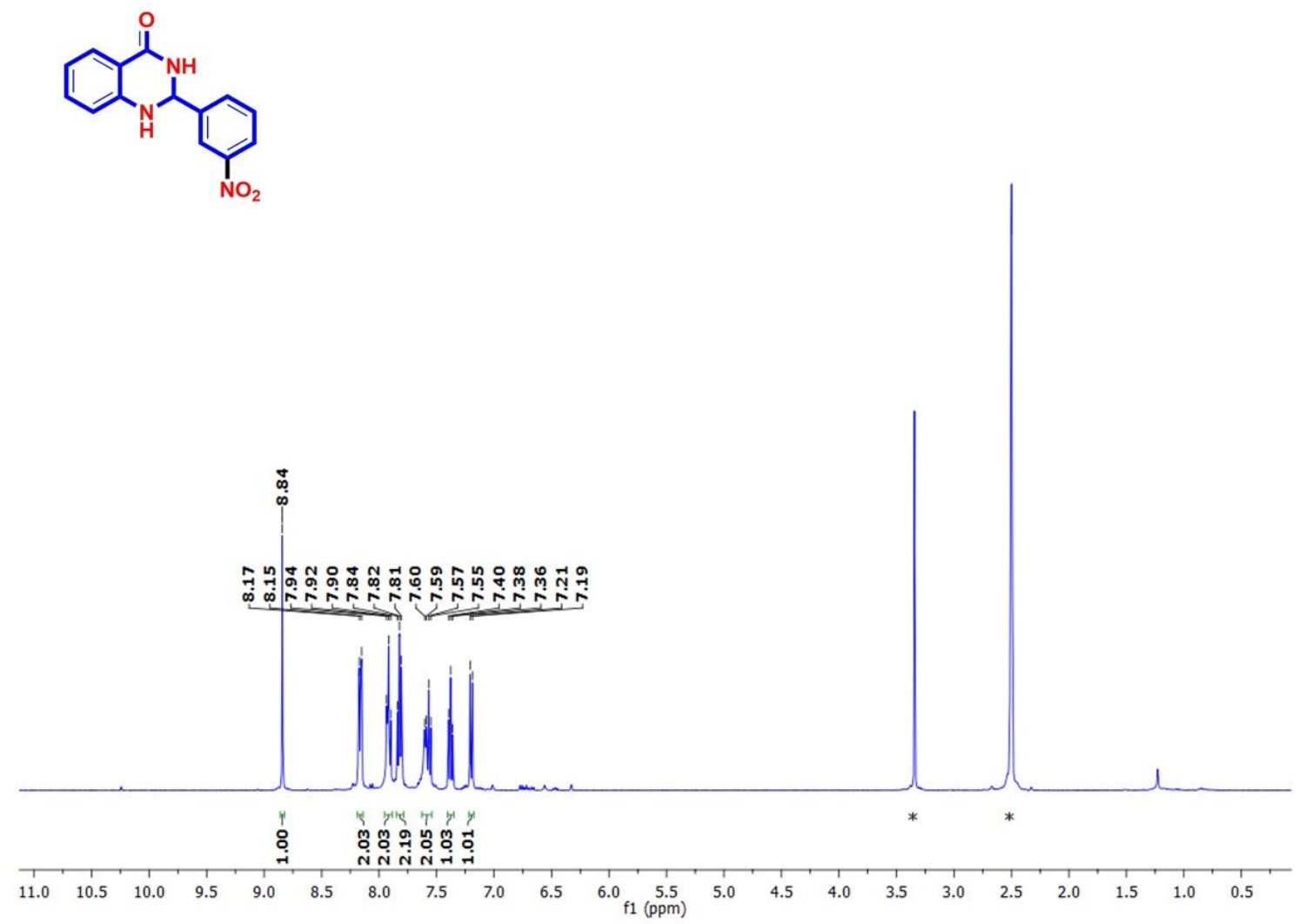

Compound G14 ${ }^{1} \mathrm{H}$ NMR
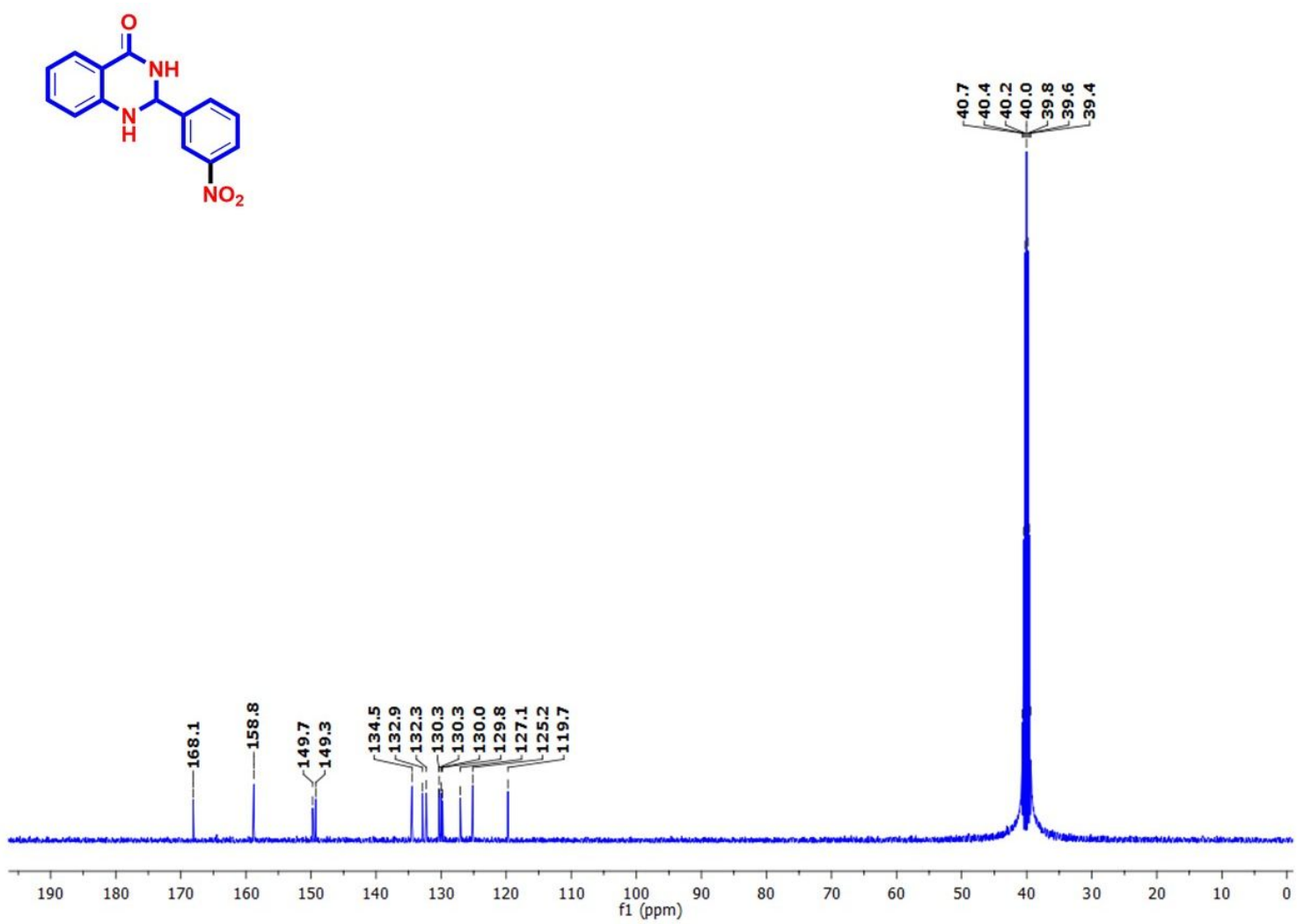

Compound G14 ${ }^{13} \mathrm{C}$ NMR 